\title{
Hybrid Iterative Scheme for Triple Hierarchical Variational Inequalities with Mixed Equilibrium, Variational Inclusion, and Minimization Constraints
}

\author{
Lu-Chuan Ceng, ${ }^{1}$ Cheng-Wen Liao, ${ }^{2}$ Chin-Tzong Pang, ${ }^{3}$ and Ching-Feng Wen ${ }^{4}$ \\ ${ }^{1}$ Department of Mathematics, Shanghai Normal University and Scientific Computing Key Laboratory of Shanghai Universities, \\ Shanghai 200234, China \\ ${ }^{2}$ Department of Food and Beverage Management, Vanung University, Chung-Li 320061, Taiwan \\ ${ }^{3}$ Department of Information Management, Innovation Center for Big Data and Digital Convergence, Yuan Ze University, \\ Chung-Li 32003, Taiwan \\ ${ }^{4}$ Center for Fundamental Science, Kaohsiung Medical University, Kaohsiung 807, Taiwan
}

Correspondence should be addressed to Chin-Tzong Pang; imctpang@saturn.yzu.edu.tw

Received 29 April 2014; Accepted 5 June 2014; Published 3 July 2014

Academic Editor: Chong Li

Copyright (c) 2014 Lu-Chuan Ceng et al. This is an open access article distributed under the Creative Commons Attribution License, which permits unrestricted use, distribution, and reproduction in any medium, provided the original work is properly cited.

\begin{abstract}
We introduce and analyze a hybrid iterative algorithm by combining Korpelevich's extragradient method, the hybrid steepestdescent method, and the averaged mapping approach to the gradient-projection algorithm. It is proven that, under appropriate assumptions, the proposed algorithm converges strongly to a common element of the fixed point set of finitely many nonexpansive mappings, the solution set of a generalized mixed equilibrium problem (GMEP), the solution set of finitely many variational inclusions, and the solution set of a convex minimization problem (CMP), which is also a unique solution of a triple hierarchical variational inequality (THVI) in a real Hilbert space. In addition, we also consider the application of the proposed algorithm to solving a hierarchical variational inequality problem with constraints of the GMEP, the CMP, and finitely many variational inclusions.
\end{abstract}

\section{Introduction}

Let $H$ be a real Hilbert space with inner product $\langle\cdot, \cdot\rangle$ and norm $\|\cdot\|$, let $C$ be a nonempty closed convex subset of $H$, and let $P_{C}$ be the metric projection of $H$ onto $C$. Let $S: C \rightarrow H$ be a nonlinear mapping on $C$. We denote by $\operatorname{Fix}(S)$ the set of fixed points of $S$ and by $\mathbf{R}$ the set of all real numbers. A mapping $S: C \rightarrow H$ is called $L$-Lipschitz continuous if there exists a constant $L>0$ such that

$$
\|S x-S y\| \leq L\|x-y\|, \quad \forall x, y \in C .
$$

In particular, if $L=1$ then $S$ is called a nonexpansive mapping; if $L \in(0,1)$ then $S$ is called a contraction.
Let $A: C \rightarrow H$ be a nonlinear mapping on $C$. We consider the following variational inequality problem (VIP) [1]: find a point $x \in C$ such that

$$
\langle A x, y-x\rangle \geq 0, \quad \forall y \in C .
$$

The solution set of VIP (2) is denoted by $\operatorname{VI}(C, A)$.

Let $\varphi: C \rightarrow \mathbf{R}$ be a real-valued function, let $A$ : $H \rightarrow H$ be a nonlinear mapping, and let $\Theta: C \times C \rightarrow \mathbf{R}$ be a bifunction. The generalized mixed equilibrium problem (GMEP) [2] is to find $x \in C$ such that

$$
\Theta(x, y)+\varphi(y)-\varphi(x)+\langle A x, y-x\rangle \geq 0, \quad \forall y \in C .
$$

We denote the set of solutions of GMEP (3) by GMEP $(\Theta, \varphi, A)$.

In [2], it is assumed that $\Theta: C \times C \rightarrow \mathbf{R}$ is a bifunction satisfying conditions (H1)-(H4) and $\varphi: C \rightarrow \mathbf{R}$ is a lower 
semicontinuous and convex function with restriction (A1) or (A2), where

(H1) $\Theta(x, x)=0$, for all $x \in C$;

$(\mathrm{H} 2) \Theta$ is monotone; that is, $\Theta(x, y)+\Theta(y, x) \leq 0$, for any $x, y \in C$;

(H3) $\Theta$ is upper-hemicontinuous; that is, for each $x, y, z \in$ C,

$$
\limsup _{t \rightarrow 0^{+}} \Theta(t z+(1-t) x, y) \leq \Theta(x, y)
$$

(H4) $\Theta(x, \cdot)$ is convex and lower semicontinuous, for each $x \in C$;

(A1) for each $x \in H$ and $r>0$, there exists a bounded subset $D_{x} \subset C$ and $y_{x} \in C$ such that, for any $z \in$ $C \backslash D_{x}$,

$$
\Theta\left(z, y_{x}\right)+\varphi\left(y_{x}\right)-\varphi(z)+\frac{1}{r}\left\langle y_{x}-z, z-x\right\rangle<0
$$

\section{(A2) $C$ is a bounded set.}

Given a positive number $r>0$, let $S_{r}^{(\Theta, \varphi)}: H \rightarrow C$ be the solution set of the auxiliary mixed equilibrium problem; that is, for each $x \in H$,

$$
\begin{aligned}
S_{r}^{(\Theta, \varphi)}(x) & \\
:=\{y \in C: & \Theta(y, z)+\varphi(z)-\varphi(y) \\
& \left.+\frac{1}{r}\left\langle K^{\prime}(y)-K^{\prime}(x), z-y\right\rangle \geq 0, \forall z \in C\right\},
\end{aligned}
$$

where $K$ is a Fréchet differential and strongly convex function on $H$. In particular, whenever $K(x)=(1 / 2)\|x\|^{2}, \forall x \in H$, $S_{r}^{(\Theta, \varphi)}$ is rewritten as $T_{r}^{(\Theta, \varphi)}$.

Let $f: C \rightarrow \mathbf{R}$ be a convex and continuously Fréchet differentiable functional. Consider the convex minimization problem (CMP) of minimizing $f$ over the constraint set $C$ :

$$
\min _{x \in C} f(x)
$$

(assuming the existence of minimizers). We denote by $\Gamma$ the set of minimizers of CMP (7).

In 2011, combining the hybrid steepest-descent method in [3], the viscosity approximation method, and averaged mapping approach to the gradient-projection algorithm (GPA) in [4], Ceng et al. [5] introduced and analyzed the following iterative algorithm:

$$
x_{n+1}=P_{C}\left[s_{n} \gamma V x_{n}+\left(I-s_{n} \mu F\right) T_{n} x_{n}\right], \quad \forall n \geq 0,
$$

where $V: C \rightarrow H$ is $l$-Lipschitzian mapping with constant $l \geq 0$ and $F: C \rightarrow H$ is a $\kappa$-Lipschitzian and $\eta$-strongly monotone operator with constants $\kappa, \eta>0$. Assume that $0<\mu<2 \eta / \kappa^{2}, 0 \leq \gamma l<\tau=1-\sqrt{1-\mu\left(2 \eta-\mu \kappa^{2}\right)}, s_{n}:=$ $s_{n}\left(\lambda_{n}\right)=\left(2-\lambda_{n} L\right) / 4$ with $\left\{\lambda_{n}\right\} \subset(0,2 / L)$ and $\lambda_{n} \rightarrow 2 / L$, and $P_{C}\left(I-\lambda_{n} \nabla f\right)=s_{n} I+\left(1-s_{n}\right) T_{n}$. Under the control conditions that (i) $s_{n} \rightarrow 0$, (ii) $\sum_{n=0}^{\infty} s_{n}=\infty$, (iii) either $\sum_{n=0}^{\infty}\left|s_{n+1}-s_{n}\right|<\infty$ or $\lim _{n \rightarrow \infty} s_{n+1} / s_{n}=1$, it was proven in [5] that the sequence $\left\{x_{n}\right\}$ generated by (8) converges strongly to some $x^{*} \in \Gamma$, which is a unique solution of the VIP

$$
\left\langle(\mu F-\gamma V) x^{*}, p-x^{*}\right\rangle \geq 0, \quad \forall p \in \Gamma .
$$

On the other hand, let $B$ be a single-valued mapping of $C$ into $H$ and let $R$ be a set-valued mapping with $D(R)=C$. Consider the following variational inclusion: find a point $x \in$ $C$ such that

$$
0 \in B x+R x
$$

We denote by $I(B, R)$ the solution set of the variational inclusion (10). Let a set-valued mapping $R: D(R) \subset H \rightarrow$ $2^{H}$ be maximal monotone. We define the resolvent operator $J_{R, \lambda}: H \rightarrow \overline{D(R)}$ associated with $R$ and $\lambda$ as follows:

$$
J_{R, \lambda}=(I+\lambda R)^{-1}, \quad \forall x \in H,
$$

where $\lambda$ is a positive number.

Let $S$ and $T$ be two nonexpansive mappings. In 2009, Yao et al. [6] considered the following hierarchical VIP: find hierarchically a fixed point of $T$, which is a solution to the VIP for monotone mapping $I-S$; namely, find $\widetilde{x} \in \operatorname{Fix}(T)$ such that

$$
\langle(I-S) \tilde{x}, p-\tilde{x}\rangle \geq 0, \quad \forall p \in \operatorname{Fix}(T) .
$$

The solution set of the hierarchical VIP (12) is denoted by $\Lambda$. It is not hard to check that solving the hierarchical VIP (12) is equivalent to solving fixed point problem of the composite mapping $P_{\mathrm{Fix}(T)} S$; that is, find $\tilde{x} \in C$ such that $\tilde{x}=P_{\mathrm{Fix}(T)} S \tilde{x}$. The authors [6] introduced and analyzed the following iterative algorithm for solving the hierarchical VIP (12):

$$
\begin{gathered}
y_{n}=\beta_{n} S x_{n}+\left(1-\beta_{n}\right) x_{n}, \\
x_{n+1}=\alpha_{n} V x_{n}+\left(1-\alpha_{n}\right) T y_{n}, \quad \forall n \geq 0 .
\end{gathered}
$$

We observed that Zeng et al. [7] introduced and considered the following triple hierarchical variational inequality (THVI).

Problem 1. Let $N$ be a positive integer. Assume that

(i) each $S_{i}: H \rightarrow H$ is a nonexpansive mapping with $\cap_{i=1}^{N} \operatorname{Fix}\left(S_{i}\right) \neq \emptyset$;

(ii) $A_{1}: H \rightarrow H$ is $\alpha$-inverse strongly monotone;

(iii) $A_{2}: H \rightarrow H$ is $\beta$-strongly monotone and $\kappa$ Lipschitz continuous;

(iv) $\operatorname{IV}\left(\cap_{i=1}^{N} \operatorname{Fix}\left(S_{i}\right), A_{1}\right) \neq \emptyset$. 
Then, the objective is to

$$
\begin{aligned}
\text { find } x^{*} \in \mathrm{VI}\left(\mathrm{VI}\left(\bigcap_{i=1}^{N} \operatorname{Fix}\left(S_{i}\right), A_{1}\right), A_{2}\right) \\
:=\left\{x^{*} \in \mathrm{VI}\left(\bigcap_{i=1}^{N} \operatorname{Fix}\left(S_{i}\right), A_{1}\right):\left\langle A_{2} x^{*}, v-x^{*}\right\rangle \geq 0,\right. \\
\left.\forall v \in \mathrm{VI}\left(\bigcap_{i=1}^{N} \operatorname{Fix}\left(S_{i}\right), A_{1}\right)\right\} .
\end{aligned}
$$

The authors [7] proposed the following algorithm for solving Problem 1.

Algorithm ZWY (see [7, Algorithm 3.2]). Let $S_{i}: H \rightarrow$ $H(i=1,2, \ldots, N)$ and $A_{j}: H \rightarrow H(j=1,2)$ satisfy assumptions (i)-(iv) in Problem 1. The following steps are presented for solving Problem 1.

Step 0. Take $\left\{\alpha_{n}\right\}_{n=0}^{\infty} \subset(0,1],\left\{\rho_{n}\right\}_{n=0}^{\infty} \subset(0,2 \alpha], \mu \in$ $\left(0,2 \beta / \kappa^{2}\right)$, choose $x_{0} \in H$ arbitrarily, and let $n:=0$.

Step 1. Given $x_{n} \in H$, compute $x_{n+1} \in H$ as

$$
\begin{gathered}
y_{n}=S_{[n+1]}\left(x_{n}-\rho_{n} A_{1} x_{n}\right), \\
x_{n+1}=y_{n}-\mu \alpha_{n} A_{2} y_{n},
\end{gathered}
$$

where $S_{[k]}:=S_{k \bmod N}$, for integer $k \geq 1$, with the mod function taking values in the set $\{1,2, \ldots, N\}$; that is, if $k=$ $j N+q$ for some integers $j \geq 0$ and $0 \leq q<N$, then $S_{[k]}=S_{N}$ if $q=0$ and $S_{[k]}=S_{q}$ if $1 \leq q<N$.

Update $n:=n+1$ and go to Step 1 .

In this paper, we introduce and study the following triple hierarchical variational inequality (THVI) with constraints of GMEP (3), CMP (7), and finitely many variational inclusions.

Problem 2. Let $M, N$ be two positive integers. Assume that

(i) $S_{i}: H \rightarrow H$ is a nonexpansive mapping, for $i=1,2, \ldots, N, A: H \rightarrow H$ is $\zeta$-inverse strongly monotone, and $f: C \rightarrow \mathbf{R}$ is a convex functional with $L$-Lipschitz continuous gradient $\nabla f$;

(ii) $\widetilde{A}_{1}: H \rightarrow H$ is $\alpha$-inverse strongly monotone and $\widetilde{A}_{2}: H \rightarrow H$ is $\beta$-strongly monotone and $\kappa$ Lipschitz continuous;

(iii) $\Theta$ is a bifunction from $C \times C$ to $\mathbf{R}$ satisfying $(\mathrm{H} 1)-(\mathrm{H} 4)$ and $\varphi: C \rightarrow \mathbf{R}$ is a lower semicontinuous and convex functional;

(iv) $R_{k}: C \rightarrow 2^{H}$ is a maximal monotone mapping and $B_{k}: C \rightarrow H$ is $\eta_{k}$-inverse strongly monotone for $k=1,2, \ldots, M$;

(v) $\operatorname{VI}\left(\Omega, \widetilde{A_{1}}\right) \neq \emptyset$, where $\Omega:=\bigcap_{i=1}^{N} \operatorname{Fix}\left(S_{i}\right) \cap$ GMEP $(\Theta, \varphi, A) \cap\left(\cap_{k=1}^{M} I\left(B_{k}, R_{k}\right)\right) \cap \Gamma$.
Then, the objective is to

$$
\begin{aligned}
& \text { find } x^{*} \in \operatorname{VI}\left(\operatorname{VI}\left(\Omega, \widetilde{A}_{1}\right), \widetilde{A_{2}}\right) \\
& :=\left\{x^{*} \in \mathrm{VI}\left(\Omega, \widetilde{A}_{1}\right):\left\langle\widetilde{A}_{2} x^{*}, v-x^{*}\right\rangle \geq 0\right. \text {, } \\
& \left.\forall v \in \operatorname{VI}\left(\Omega, \widetilde{A_{1}}\right)\right\} .
\end{aligned}
$$

Motivated and inspired by the above facts, we introduce and analyze a hybrid iterative algorithm by combining Korpelevich's extragradient method, the hybrid steepest-descent method, and the averaged mapping approach to the gradientprojection algorithm. It is proven that under mild conditions, the proposed algorithm converges strongly to a common element $x^{*} \in \Omega:=\left(\cap_{i=1}^{N} \operatorname{Fix}\left(S_{i}\right)\right) \cap \operatorname{GMEP}(\Theta, \varphi, A) \cap$ $\left(\cap_{k=1}^{M} I\left(B_{k}, R_{k}\right)\right) \cap \Gamma$ of the solution set of GMEP (3), the solution set of CMP (7), the solution set of finitely many variational inclusions, and the fixed point set of finitely many nonexpansive mappings $\left\{S_{i}\right\}_{i=1}^{N}$, which is merely a unique solution of the THVI (16). In addition, we also consider the application of the proposed algorithm to solving a hierarchical variational inequality problem with constraints of GMEP (3), CMP (7), and finitely many variational inclusions. That is, under appropriate conditions, it is proven that the proposed algorithm converges strongly to a unique solution $u^{*} \in \Omega$ of the VIP: $\left\langle\widetilde{A}_{2} u^{*}, p-u^{*}\right\rangle \geq 0, \forall p \in \Omega$; equivalently, $P_{\Omega}\left(I-\widetilde{A_{2}}\right) u^{*}=u^{*}$. The results obtained in this paper improve and extend the corresponding results announced by many others. We also observe that some recent and related results have been established in [8-14].

\section{Preliminaries}

Throughout this paper, we assume that $H$ is a real Hilbert space whose inner product and norm are denoted by $\langle\cdot, \cdot\rangle$ and $\|\cdot\|$, respectively. Let $C$ be a nonempty closed convex subset of $H$. We write $x_{n} \rightarrow x$ to indicate that the sequence $\left\{x_{n}\right\}$ converges weakly to $x$ and $x_{n} \rightarrow x$ to indicate that the sequence $\left\{x_{n}\right\}$ converges strongly to $x$. Moreover, we use $\omega_{w}\left(x_{n}\right)$ to denote the weak $\omega$-limit set of the sequence $\left\{x_{n}\right\}$; that is,

$$
\begin{aligned}
& \omega_{w}\left(x_{n}\right):=\left\{x \in H: x_{n_{i}} \rightarrow x\right. \\
&\left.\quad \text { for some subsequence }\left\{x_{n_{i}}\right\} \text { of }\left\{x_{n}\right\}\right\} .
\end{aligned}
$$

Definition 3. A mapping $A: C \rightarrow H$ is called

(i) monotone if

$$
\langle A x-A y, x-y\rangle \geq 0, \quad \forall x, y \in C ;
$$

(ii) $\eta$-strongly monotone if there exists a constant $\eta>0$ such that

$$
\langle A x-A y, x-y\rangle \geq \eta\|x-y\|^{2}, \quad \forall x, y \in C ;
$$

(iii) $\zeta$-inverse strongly monotone if there exists a constant $\zeta>0$ such that

$$
\langle A x-A y, x-y\rangle \geq \zeta\|A x-A y\|^{2}, \quad \forall x, y \in C .
$$


It is obvious that if $A$ is $\zeta$-inverse strongly monotone, then $A$ is monotone and $1 / \zeta$-Lipschitz continuous. Moreover, we also have that, for all $u, v \in C$ and $\lambda>0$,

$$
\begin{aligned}
\|(I- & \lambda A) u-(I-\lambda A) v \|^{2} \\
& \leq\|u-v\|^{2}+\lambda(\lambda-2 \zeta)\|A u-A v\|^{2} .
\end{aligned}
$$

So, if $\lambda \leq 2 \zeta$, then $I-\lambda A$ is a nonexpansive mapping from $C$ to $H$.

Definition 4. A differentiable function $K: H \rightarrow \mathbf{R}$ is called

(i) convex, if

$$
K(y)-K(x) \geq\left\langle K^{\prime}(x), y-x\right\rangle, \quad \forall x, y \in H,
$$

where $K^{\prime}(x)$ is the Frechet derivative of $K$ at $x$;

(ii) strongly convex, if there exists a constant $\sigma>0$ such that

$$
K(y)-K(x)-\left\langle K^{\prime}(x), y-x\right\rangle \geq \frac{\sigma}{2}\|x-y\|^{2}, \quad \forall x, y \in H .
$$

It is easy to see that if $K: H \rightarrow \mathbf{R}$ is a differentiable strongly convex function with a constant $\sigma>0$ then $K^{\prime}$ : $H \rightarrow H$ is strongly monotone with constant $\sigma>0$.

The metric projection from $H$ onto $C$ is the mapping $P_{C}$ : $H \rightarrow C$ which assigns to each point $x \in H$ the unique point $P_{C} x \in C$ satisfying the property

$$
\left\|x-P_{C} x\right\|=\inf _{y \in C}\|x-y\|=: d(x, C) .
$$

Some important properties of projections are gathered in the following proposition.

Proposition 5. For given $x \in H$ and $z \in C$ :

(i) $z=P_{C} x \Leftrightarrow\langle x-z, y-z\rangle \leq 0, \forall y \in C$;

(ii) $z=P_{C} x \Leftrightarrow\|x-z\|^{2} \leq\|x-y\|^{2}-\|y-z\|^{2}, \forall y \in C$;

(iii) $\left\langle P_{C} x-P_{C} y, x-y\right\rangle \geq\left\|P_{C} x-P_{C} y\right\|^{2}, \forall y \in H$ (this implies that $P_{C}$ is nonexpansive and monotone).

By using the technique of [15], we can readily obtain the following elementary result, where $\operatorname{MEP}(\Theta, \varphi)$ is the solution set of the mixed equilibrium problem [15].

Proposition 6 (see [16, Lemma 1 and Proposition 1]). Let $C$ be a nonempty closed convex subset of a real Hilbert space $H$ and let $\varphi: C \rightarrow \mathbf{R}$ be a lower semicontinuous and convex function. Let $\Theta: C \times C \rightarrow \mathbf{R}$ be a bifunction satisfying the conditions (H1)-(H4). Assume that

(i) $\mathrm{K}: H \rightarrow \mathbf{R}$ is strongly convex with constant $\sigma>0$ and the function $x \mapsto\left\langle y-x, K^{\prime}(x)\right\rangle$ is weakly upper semicontinuous, for each $y \in H$; (ii) for each $x \in H$ and $r>0$ there exists a bounded subset $D_{x} \subset C$ and $y_{x} \in C$ such that, for any $z \in C \backslash D_{x}$,

$\Theta\left(z, y_{x}\right)+\varphi\left(y_{x}\right)-\varphi(z)+\frac{1}{r}\left\langle K^{\prime}(z)-K^{\prime}(x), y_{x}-z\right\rangle<0$.

Then, the following hold:

(a) for each $x \in H, S_{r}^{(\Theta, \varphi)}(x) \neq \emptyset$;

(b) $S_{r}^{(\Theta, \varphi)}$ is single-valued;

(c) $S_{r}^{(\Theta, \varphi)}$ is nonexpansive if $K^{\prime}$ is Lipschitz continuous with constant $\nu>0$ and

$$
\begin{aligned}
& \left\langle K^{\prime}\left(x_{1}\right)-K^{\prime}\left(x_{2}\right), u_{1}-u_{2}\right\rangle \\
& \quad \leq\left\langle K^{\prime}\left(u_{1}\right)-K^{\prime}\left(u_{2}\right), u_{1}-u_{2}\right\rangle, \quad \forall\left(x_{1}, x_{2}\right) \in H \times H,
\end{aligned}
$$

$$
\text { where } u_{i}=S_{r}^{(\Theta, \varphi)}\left(x_{i}\right), \text { for } i=1,2 ;
$$

(d) for all $s, t>0$ and $x \in H$,

$$
\begin{aligned}
& \left\langle K^{\prime}\left(S_{s}^{(\Theta, \varphi)} x\right)-K^{\prime}\left(S_{t}^{(\Theta, \varphi)} x\right), S_{s}^{(\Theta, \varphi)} x-S_{t}^{(\Theta, \varphi)} x\right\rangle \\
& \quad \leq \frac{s-t}{s}\left\langle K^{\prime}\left(S_{s}^{(\Theta, \varphi)} x\right)-K^{\prime}(x), S_{s}^{(\Theta, \varphi)} x-S_{t}^{(\Theta, \varphi)} x\right\rangle ;
\end{aligned}
$$

(e) $\operatorname{Fix}\left(S_{r}^{(\Theta, \varphi)}\right)=\operatorname{MEP}(\Theta, \varphi)$;

(f) $\operatorname{MEP}(\Theta, \varphi)$ is closed and convex.

In particular, whenever $\Theta: C \times C \rightarrow \mathbf{R}$ is a bifunction satisfying the conditions (H1)-(H4) and $K(x)=(1 / 2)\|x\|^{2}, \forall x \in H$, then we have that $S_{r}^{(\Theta, \varphi)}$ is firmly nonexpansive and

$$
\begin{array}{r}
\left\|S_{s}^{(\Theta, \varphi)} x-S_{t}^{(\Theta, \varphi)} x\right\| \leq \frac{|s-t|}{s}\left\|S_{s}^{(\Theta, \varphi)} x-x\right\|, \\
\forall s, t>0, \quad x \in H .
\end{array}
$$

In this case, $S_{r}^{(\Theta, \varphi)}$ is rewritten as $T_{r}^{(\Theta, \varphi)}$. If, in addition, $\varphi \equiv 0$, then $T_{r}^{(\Theta, \varphi)}$ is rewritten as $T_{r}^{\Theta}$ (see [17, Lemma 2.1] for more details).

Remark 7. Suppose that $K: H \rightarrow \mathbf{R}$ is strongly convex with constant $\sigma>0$ and $K^{\prime}: H \rightarrow H$ is Lipschitz continuous with constant $\nu>0$. Then, $K^{\prime}: H \rightarrow H$ is $\sigma$-strongly monotone and $\nu$-Lipschitz continuous with positive constants $\sigma, \nu>0$. Utilizing Proposition 6 (d) we obtain that, for all $s, t>0$ and $x \in H$,

$$
\begin{aligned}
\sigma \| & S_{s}^{(\Theta, \varphi)} x-S_{t}^{(\Theta, \varphi)} x \|^{2} \\
& \leq\left\langle K^{\prime}\left(S_{s}^{(\Theta, \varphi)} x\right)-K^{\prime}\left(S_{t}^{(\Theta, \varphi)} x\right), S_{s}^{(\Theta, \varphi)} x-S_{t}^{(\Theta, \varphi)} x\right\rangle \\
& \leq \frac{s-t}{s}\left\langle K^{\prime}\left(S_{s}^{(\Theta, \varphi)} x\right)-K^{\prime}(x), S_{s}^{(\Theta, \varphi)} x-S_{t}^{(\Theta, \varphi)} x\right\rangle \\
& \leq \frac{|s-t|}{s}\left\|K^{\prime}\left(S_{s}^{(\Theta, \varphi)} x\right)-K^{\prime}(x)\right\|\left\|S_{s}^{(\Theta, \varphi)} x-S_{t}^{(\Theta, \varphi)} x\right\| \\
& \leq \frac{|s-t|}{s} v\left\|S_{s}^{(\Theta, \varphi)} x-x\right\|\left\|S_{s}^{(\Theta, \varphi)} x-S_{t}^{(\Theta, \varphi)} x\right\|,
\end{aligned}
$$


which immediately implies that

$$
\left\|S_{s}^{(\Theta, \varphi)} x-S_{t}^{(\Theta, \varphi)} x\right\| \leq \frac{|s-t|}{s} \cdot \frac{v}{\sigma}\left\|S_{s}^{(\Theta, \varphi)} x-x\right\| .
$$

The concept of $W$-mapping was introduced in Atsushiba and Takahashi [18]. It is very useful in establishing the convergence of iterative methods for computing a common fixed point of nonlinear mappings (see, for instance, $[19,20]$ ).

Let $\mu_{1, n}, \mu_{2, n}, \ldots, \mu_{N, n} \in(0,1], n \geq 1$. Given the nonexpansive mappings $S_{1}, S_{2}, \ldots, S_{N}$ on $C$, Atsushiba and Takahashi define, for each $n \geq 1$, mappings $U_{1, n}, U_{2, n}, \ldots, U_{N, n}$, by

$$
\begin{aligned}
& U_{1, n}=\mu_{1, n} S_{1}+\left(1-\mu_{1, n}\right) I, \\
& U_{2, n}=\mu_{2, n} S_{2}+\left(1-\mu_{2, n}\right) I, \\
& \vdots \\
& U_{N-1, n}=\mu_{N-1, n} S_{N-1} U_{N-2, n}+\left(1-\mu_{N-1, n}\right) I, \\
& W_{n}:=U_{N, n}=\mu_{N, n} S_{N} U_{N-1, n}+\left(1-\mu_{N, n}\right) I .
\end{aligned}
$$

The $W_{n}$ is called the $W$-mapping generated by $S_{1}$, $S_{2}, \ldots, S_{N}$ and $\mu_{1, n}, \mu_{2, n}, \ldots, \mu_{N, n}$. Note that the nonexpansivity of $S_{i}$ implies the one of $W_{n}$.

Proposition 8 (see [20]). Let $C$ be a nonempty closed convex subset of a Banach space $X$. Let $S_{1}, S_{2}, \ldots, S_{N}$ be a finite family of nonexpansive mappings of $C$ into itself such that $\cap_{i=1}^{N} \operatorname{Fix}\left(S_{i}\right) \neq \emptyset$, and let $\mu_{1, n}, \mu_{2, n}, \ldots, \mu_{N, n}$ be real numbers such that $0<\mu_{i, n} \leq b<1$ for $i=1,2, \ldots, N$. For any $n \geq 1$, let $W_{n}$ be the $W$-mapping of $C$ into itself generated by $S_{1}, S_{2}, \ldots, S_{N}$ and $\mu_{1, n}, \mu_{2, n}, \ldots, \mu_{N, n}$. If $X$ is strictly convex, then $\operatorname{Fix}\left(W_{n}\right)=$ $\cap_{i=1}^{N} \operatorname{Fix}\left(S_{i}\right)$.

Proposition 9 (see [21, Lemma 2.8]). Let $C$ be a nonempty convex subset of a Banach space X. Let $\left\{S_{i}\right\}_{i=1}^{N}$ be a finite family of nonexpansive mappings of $C$ into itself and let $\left\{\mu_{i, n}\right\}_{i=1}^{N}$ be sequences in $[0,1]$ such that $\mu_{i, n} \rightarrow \mu_{i}(i=1,2, \ldots, N)$. Moreover, for every integer $n \geq 1$, let $W$ and $W_{n}$ be the $W$ mapping generated by $S_{1}, S_{2}, \ldots, S_{N}$ and $\mu_{1}, \mu_{2}, \ldots, \mu_{N}$ and $S_{1}, S_{2}, \ldots, S_{N}$ and $\mu_{1, n}, \mu_{2, n}, \ldots, \mu_{N, n}$, respectively. Then, for every $x \in C$, it follows that

$$
\lim _{n \rightarrow \infty}\left\|W_{n} x-W x\right\|=0 .
$$

In what follows, we recall some facts and tools in a real Hilbert space $H$.

Lemma 10. Let $X$ be a real inner product space. Then, the following inequality holds:

$$
\|x+y\|^{2} \leq\|x\|^{2}+2\langle y, x+y\rangle, \quad \forall x, y \in X .
$$

Lemma 11. Let $H$ be a real Hilbert space. Then, the following hold:

(a) $\|x-y\|^{2}=\|x\|^{2}-\|y\|^{2}-2\langle x-y, y\rangle$, for all $x, y \in H$;

(b) $\|\lambda x+\mu y\|^{2}=\lambda\|x\|^{2}+\mu\|y\|^{2}-\lambda \mu\|x-y\|^{2}$, for all $x, y \in$ $H$ and $\lambda, \mu \in[0,1]$ with $\lambda+\mu=1$; (c) If $\left\{x_{n}\right\}$ is a sequence in $H$ such that $x_{n} \rightarrow x$, it follows that

$\limsup _{n \rightarrow \infty}\left\|x_{n}-y\right\|^{2}=\limsup _{n \rightarrow \infty}\left\|x_{n}-x\right\|^{2}+\|x-y\|^{2}, \quad \forall y \in H$.

Definition 12. A mapping $T: H \rightarrow H$ is said to be an averaged mapping if it can be written as the average of the identity $I$ and a nonexpansive mapping; that is,

$$
T \equiv(1-\alpha) I+\alpha S
$$

where $\alpha \in(0,1)$ and $S: H \rightarrow H$ is nonexpansive. More precisely, when the last equality holds, we say that $T$ is $\alpha$ averaged. Thus, firmly nonexpansive mappings (in particular, projections) are $1 / 2$-averaged mappings.

Lemma 13 (see [22]). Let $T: H \rightarrow H$ be a given mapping.

(i) $T$ is nonexpansive if and only if the complement $I-T$ is $1 / 2$-ism.

(ii) If $T$ is $\nu$-ism, then, for $\gamma>0, \gamma T$ is $\nu / \gamma$-ism.

(iii) $T$ is averaged if and only if the complement $I-T$ is $\nu$-ism for some $\nu>1 / 2$. Indeed, for $\alpha \in(0,1), T$ is $\alpha$-averaged if and only if $I-T$ is $1 / 2 \alpha$-ism.

Lemma 14 (see [22]). Let $S, T, V: H \rightarrow H$ be given operators.

(i) If $T=(1-\alpha) S+\alpha V$ for some $\alpha \in(0,1)$ and if $S$ is averaged and $V$ is nonexpansive, then $T$ is averaged.

(ii) $T$ is firmly nonexpansive if and only if the complement $I-T$ is firmly nonexpansive.

(iii) If $T=(1-\alpha) S+\alpha V$ for some $\alpha \in(0,1)$ and if $S$ is firmly nonexpansive and $V$ is nonexpansive, then $T$ is averaged.

(iv) The composite of finitely many averaged mappings is averaged. That is, if each of the mappings $\left\{T_{i}\right\}_{i=1}^{N}$ is averaged, then so is the composite $T_{1} \cdots T_{N}$. In particular, if $T_{1}$ is $\alpha_{1}$-averaged and $T_{2}$ is $\alpha_{2}$-averaged, where $\alpha_{1}, \alpha_{2} \in(0,1)$, then the composite $T_{1} T_{2}$ is $\alpha$ averaged, where $\alpha=\alpha_{1}+\alpha_{2}-\alpha_{1} \alpha_{2}$.

(v) If the mappings $\left\{T_{i}\right\}_{i=1}^{N}$ are averaged and have a common fixed point, then

$$
\bigcap_{i=1}^{N} \operatorname{Fix}\left(T_{i}\right)=\operatorname{Fix}\left(T_{1} T_{2} \cdots T_{N}\right) .
$$

The notation $\operatorname{Fix}(T)$ denotes the set of all fixed points of the mapping T; that is, $\operatorname{Fix}(T)=\{x \in H: T x=x\}$.

Let $f: C \rightarrow \mathbf{R}$ be a convex functional with $L$ Lipschitz continuous gradient $\nabla f$. It is well known that the gradient-projection algorithm (GPA) generates a sequence $\left\{x_{n}\right\}$ determined by the gradient $\nabla f$ and the metric projection $P_{C}$ :

$$
x_{n+1}:=P_{C}\left(x_{n}-\lambda \nabla f\left(x_{n}\right)\right), \quad \forall n \geq 0,
$$


or, more generally,

$$
x_{n+1}:=P_{C}\left(x_{n}-\lambda_{n} \nabla f\left(x_{n}\right)\right), \quad \forall n \geq 0,
$$

where, in both (37) and (38), the initial guess $x_{0}$ is taken from $C$ arbitrarily and the parameters $\lambda$ or $\lambda_{n}$ are positive real numbers. The convergence of algorithms (37) and (38) depends on the behavior of the gradient $\nabla f$.

Lemma 15 (see [23, demiclosedness principle]). Let $C$ be a nonempty closed convex subset of a real Hilbert space $H$. Let $T$ be a nonexpansive self-mapping on $C$. Then, $I-T$ is demiclosed. That is, whenever $\left\{x_{n}\right\}$ is a sequence in $C$ weakly converging to some $x \in C$ and the sequence $\left\{(I-T) x_{n}\right\}$ strongly converges to some $y$, it follows that $(I-T) x=y$. Here $I$ is the identity operator of $H$.

Lemma 16. Let $A: C \rightarrow H$ be a monotone mapping. In the context of the variational inequality problem the characterization of the projection (see Proposition 5 (i)) implies

$$
u \in \mathrm{VI}(C, A) \Longleftrightarrow u=P_{C}(u-\lambda A u), \quad \lambda>0 .
$$

Let $C$ be a nonempty closed convex subset of a real Hilbert space $H$. We introduce some notations. Let $\lambda$ be a number in $(0,1]$ and let $\mu>0$. Associating with a nonexpansive mapping $T: C \rightarrow H$, we define the mapping $T^{\lambda}: C \rightarrow H$ by

$$
T^{\lambda} x:=T x-\lambda \mu F(T x), \quad \forall x \in C,
$$

where $F: H \rightarrow H$ is an operator such that, for some positive constants $\kappa, \eta>0, F$ is $\kappa$-Lipschitzian and $\eta$-strongly monotone on $H$; that is, $F$ satisfies the conditions

$$
\|F x-F y\| \leq \kappa\|x-y\|, \quad\langle F x-F y, x-y\rangle \geq \eta\|x-y\|^{2}
$$

for all $x, y \in H$.

Lemma 17 (see [3, Lemma 3.1]). $T^{\lambda}$ is a contraction provided $0<\mu<2 \eta / \kappa^{2}$; that is,

$$
\left\|T^{\lambda} x-T^{\lambda} y\right\| \leq(1-\lambda \tau)\|x-y\|, \quad \forall x, y \in C,
$$

where $\tau=1-\sqrt{1-\mu\left(2 \eta-\mu \kappa^{2}\right)} \in(0,1]$.

Lemma 18 (see [3]). Let $\left\{s_{n}\right\}$ be a sequence of nonnegative numbers satisfying the conditions

$$
s_{n+1} \leq\left(1-\alpha_{n}\right) s_{n}+\alpha_{n} \beta_{n}, \quad \forall n \geq 1,
$$

where $\left\{\alpha_{n}\right\}$ and $\left\{\beta_{n}\right\}$ are sequences of real numbers such that

(i) $\left\{\alpha_{n}\right\} \subset[0,1]$ and $\sum_{n=1}^{\infty} \alpha_{n}=\infty$ or, equivalently,

$$
\prod_{n=1}^{\infty}\left(1-\alpha_{n}\right):=\lim _{n \rightarrow \infty} \prod_{k=1}^{n}\left(1-\alpha_{k}\right)=0 ;
$$

(ii) $\lim \sup _{n \rightarrow \infty} \beta_{n} \leq 0$, or $\sum_{n=1}^{\infty}\left|\alpha_{n} \beta_{n}\right|<\infty$.

Then, $\lim _{n \rightarrow \infty} s_{n}=0$.
Recall that a Banach space $X$ is said to satisfy Opial's property [23] if for any given sequence $\left\{x_{n}\right\} \subset X$ which converges weakly to an element $x \in X$, there holds the inequality

$$
\limsup _{n \rightarrow \infty}\left\|x_{n}-x\right\|<\limsup _{n \rightarrow \infty}\left\|x_{n}-y\right\|, \quad \forall y \in X, \quad y \neq x .
$$

It is well known that every Hilbert space $H$ satisfies Opial's property in [23].

Finally, recall that a set-valued mapping $T: D(T) \subset H \rightarrow$ $2^{H}$ is called monotone if for all $x, y \in D(T), f \in T x$ and $g \in T y$ imply

$$
\langle f-g, x-y\rangle \geq 0
$$

A set-valued mapping $T$ is called maximal monotone if $T$ is monotone and $(I+\lambda T) D(T)=H$, for each $\lambda>0$, where $I$ is the identity mapping of $H$. We denote by $G(T)$ the graph of $T$. It is known that a monotone mapping $T$ is maximal if and only if, for $(x, f) \in H \times H,\langle f-g, x-y\rangle \geq 0$ for every $(y, g) \in G(T)$ implies $f \in T x$. Let $A: C \rightarrow H$ be a monotone, $k$-Lipschitzcontinuous mapping, and let $N_{C} v$ be the normal cone to $C$ at $v \in C$; that is,

$$
N_{C} v=\{u \in H:\langle v-p, u\rangle \geq 0, \forall p \in C\} .
$$

Define

$$
\widetilde{T} v= \begin{cases}A v+N_{C} v, & \text { if } v \in C, \\ \emptyset, & \text { if } v \notin C .\end{cases}
$$

Then, $\widetilde{T}$ is maximal monotone and

$$
0 \in \widetilde{T} v \Longleftrightarrow v \in \mathrm{VI}(C, A) .
$$

Let $R: D(R) \subset H \rightarrow 2^{H}$ be a maximal monotone mapping. Let $\lambda, \mu>0$ be two positive numbers.

Lemma 19 (see [24]). There holds the resolvent identity

$$
J_{R, \lambda} x=J_{R, \mu}\left(\frac{\mu}{\lambda} x+\left(1-\frac{\mu}{\lambda}\right) J_{R, \lambda} x\right), \quad \forall x \in H .
$$

For $\lambda, \mu>0$, we observe that there holds the following relation:

$$
\begin{aligned}
& \left\|J_{R, \lambda} x-J_{R, \mu} y\right\| \leq\|x-y\| \\
& \quad+|\lambda-\mu|\left(\frac{1}{\lambda}\left\|J_{R, \lambda} x-y\right\|+\frac{1}{\mu}\left\|x-J_{R, \mu} y\right\|\right), \quad \forall x, y \in H .
\end{aligned}
$$

Lemma 20 (see [25]). $J_{R, \lambda}$ is single-valued and firmly nonexpansive; that is,

$$
\left\langle J_{R, \lambda} x-J_{R, \lambda} y, x-y\right\rangle \geq\left\|J_{R, \lambda} x-J_{R, \lambda} y\right\|^{2}, \quad \forall x, y \in H .
$$

Consequently, $J_{R, \lambda}$ is nonexpansive and monotone. 
Lemma 21 (see [26]). Let $R$ be a maximal monotone mapping with $D(R)=C$. Then, for any given $\lambda>0, u \in C$ is a solution of problem (11) if and only if $u \in C$ satisfies

$$
u=J_{R, \lambda}(u-\lambda B u)
$$

Lemma 22 (see [27]). Let $R$ be a maximal monotone mapping with $D(R)=C$ and let $B: C \rightarrow H$ be a strongly monotone, continuous, and single-valued mapping. Then, for each $z \in H$, the equation $z \in(B+\lambda R) x$ has a unique solution $x_{\lambda}$ for $\lambda>0$.

Lemma 23 (see [26]). Let $R$ be a maximal monotone mapping with $D(R)=C$ and let $B: C \rightarrow H$ be a monotone, continuous, and single-valued mapping. Then, $(I+\lambda(R+B)) C=H$ for each $\lambda>0$. In this case, $R+B$ is maximal monotone.

\section{Main Results}

In this section, we will introduce and analyze a hybrid iterative algorithm for finding a solution of the THVI (16) with constraints of several problems: the GMEP (3), the CMP (7), and finitely many variational inclusions in a real Hilbert space. This algorithm is based on Korpelevich's extragradient method, hybrid steepest-descent method, and averaged mapping approach to the gradient-projection algorithm. We prove the strong convergence of the proposed algorithm to a unique solution of THVI (16) under suitable conditions. In addition, we also consider the application of the proposed algorithm to solving a hierarchical VIP with the same constraints.

We are now in a position to state and prove the first main result in this paper.

Theorem 24. Let $C$ be a nonempty closed convex subset of a real Hilbert space $H$. Let $f: C \rightarrow \mathbf{R}$ be a convex functional with L-Lipschitz continuous gradient $\nabla f$. Let $N, N \geq 1$ be two integers. Let $\Theta$ be a bifunction from $C \times C$ to $\mathbf{R}$ satisfying (H1)-(H4), let $\varphi: C \rightarrow \mathbf{R}$ be a lower semicontinuous and convex functional, and let $A: H \rightarrow H$ be $\zeta$-inverse strongly monotone. Let $R_{k}: C \rightarrow 2^{H}$ be a maximal monotone mapping and let $B_{k}: C \rightarrow H$ be $\eta_{k}$-inverse strongly monotone for $k=1,2, \ldots, M$. Let $\left\{S_{i}\right\}_{i=1}^{N}$ be a finite family of nonexpansive mappings on $H$. Let $\widetilde{A}_{1}: H \rightarrow H$ be $\alpha$-inverse strongly monotone and let $\widetilde{A}_{2}: H \rightarrow H$ be $\beta$-strongly monotone and $\kappa$-Lipschitz continuous. Assume that $\operatorname{VI}\left(\Omega, \widetilde{A}_{1}\right) \neq \emptyset$, where $\Omega:=\cap_{i=1}^{N} \operatorname{Fix}\left(S_{i}\right) \cap \operatorname{GMEP}(\Theta, \varphi, A) \cap \cap_{k=1}^{M} I\left(B_{k}, R_{k}\right) \cap \Gamma$. Let $\mu \in\left(0,2 \beta / \kappa^{2}\right),\left\{\alpha_{n}\right\}_{n=1}^{\infty} \subset(0,1],\left\{\rho_{n}\right\}_{n=1}^{\infty} \subset(0,2 \alpha],\left\{\beta_{n}\right\}_{n=1}^{\infty} \subset$ $[a, b] \subset(0,1),\left\{r_{n}\right\}_{n=1}^{\infty} \subset[c, d] \subset(0,2 \zeta),\left\{\mu_{i, n}\right\}_{n=1}^{\infty} \subset[e, f] \subset$ $(0,1),\left\{\lambda_{k, n}\right\}_{n=1}^{\infty} \subset\left[a_{k}, b_{k}\right] \subset\left(0,2 \eta_{k}\right)$, where $k \in\{1,2, \ldots, M\}$ and $i \in\{1,2, \ldots, N\}$. For every $n \geq 1$, let $W_{n}$ be the $W$-mapping generated by $S_{1}, S_{2}, \ldots, S_{N}$ and $\mu_{1, n}, \mu_{2, n}, \ldots, \mu_{N, n}$. Assume that

(i) $K: H \rightarrow \mathbf{R}$ is strongly convex with a constant $\sigma>0$ and its derivative $K^{\prime}$ is Lipschitz continuous with a constant $v>0$ such that the function $x \mapsto$ $\left\langle y-x, K^{\prime}(x)\right\rangle$ is weakly upper semicontinuous for each $y \in H$; (ii) for each $x \in H$, there exist a bounded subset $D_{x} \subset C$ and $z_{x} \in C$ such that, for any $y \notin D_{x}$,

$\Theta\left(y, z_{x}\right)+\varphi\left(z_{x}\right)-\varphi(y)+\frac{1}{r}\left\langle K^{\prime}(y)-K^{\prime}(x), z_{x}-y\right\rangle<0 ;$

(iii) $\lim _{n \rightarrow \infty} \alpha_{n}=0, \sum_{n=1}^{\infty} \alpha_{n}=\infty$ and $\lim _{n \rightarrow \infty}\left(1 / \alpha_{n}\right) \mid 1-$ $\left(\rho_{n-1} / \rho_{n}\right) \mid=0$

(iv) $\lim _{n \rightarrow \infty}\left(\rho_{n} / \alpha_{n}\right)=0, \lim _{n \rightarrow \infty}\left(1 / \alpha_{n}\right) \mid\left(1 / \rho_{n}\right)-$ $\left(1 / \rho_{n-1}\right) \mid=0$, and $\lim _{n \rightarrow \infty}\left(1 / \rho_{n}\right)\left|1-\left(\alpha_{n-1} / \alpha_{n}\right)\right|=0$;

(v) $\lim _{n \rightarrow \infty}\left(\left|\beta_{n}-\beta_{n-1}\right| / \alpha_{n} \rho_{n}\right)=0$ and $\lim _{n \rightarrow \infty}\left(\mid \lambda_{k, n}-\right.$ $\left.\lambda_{k, n-1} \mid / \alpha_{n} \rho_{n}\right)=0$, for $k=1,2, \ldots, M$;

(vi) $\lim _{n \rightarrow \infty}\left(\left|r_{n}-r_{n-1}\right| / \alpha_{n} \rho_{n}\right)=0$ and $\lim _{n \rightarrow \infty}\left(\mid \mu_{i, n}-\right.$ $\left.\mu_{i, n-1} \mid / \alpha_{n} \rho_{n}\right)=0$, for $i=1,2, \ldots, N$.

For arbitrarily given $x_{1} \in H$, let $\left\{x_{n}\right\}$ be a sequence generated by

$$
\begin{gathered}
u_{n}=S_{r_{n}}^{(\Theta, \varphi)}\left(I-r_{n} A\right) x_{n} \\
v_{n}=J_{R_{M}, \lambda_{M, n}}\left(I-\lambda_{M, n} B_{M}\right) J_{R_{M-1}, \lambda_{M-1, n}} \\
\times\left(I-\lambda_{M-1, n} B_{M-1}\right) \cdots J_{R_{1}, \lambda_{1, n}}\left(I-\lambda_{1, n} B_{1}\right) u_{n}, \\
y_{n}=\beta_{n} T_{n} v_{n}+\left(1-\beta_{n}\right) W_{n}\left(v_{n}-\rho_{n} \widetilde{A}_{1} v_{n}\right), \\
x_{n+1}=y_{n}-\mu \alpha_{n} \widetilde{A}_{2} y_{n}, \quad \forall n \geq 1,
\end{gathered}
$$

where $P_{C}\left(I-\lambda_{n} \nabla f\right)=s_{n} I+\left(1-s_{n}\right) T_{n}$ (here $T_{n}$ is nonexpansive, $s_{n}=\left(2-\lambda_{n} L\right) / 4 \in(0,1 / 2)$ for each $\left.\lambda_{n} \in(0,2 / L)\right)$, $\lim _{n \rightarrow \infty} s_{n}=0\left(\Leftrightarrow \lim _{n \rightarrow \infty} \lambda_{n}=2 / L\right)$, and $\lim _{n \rightarrow \infty}\left(\mid s_{n}-\right.$ $\left.s_{n-1} \mid / \alpha_{n} \rho_{n}\right)=0$. Then, whenever $S_{r}^{(\Theta, \varphi)}$ is firmly nonexpansive, the following hold:

(i) $\lim _{n \rightarrow \infty}\left(\left\|x_{n+1}-x_{n}\right\| / \rho_{n}\right)=0$;

(ii) $\omega_{w}\left(x_{n}\right) \subset \Omega$;

(iii) $\omega_{w}\left(x_{n}\right) \subset \operatorname{VI}\left(\Omega, \widetilde{A}_{1}\right)$ provided $\left\|x_{n}-y_{n}\right\|=o\left(\rho_{n}\right)$ additionally.

Proof. Let $\left\{x^{*}\right\}=\operatorname{VI}\left(\operatorname{VI}\left(\Omega, \widetilde{A}_{1}\right), \widetilde{A}_{2}\right)$. Taking into account that $\lim _{n \rightarrow \infty}\left(\rho_{n} / \alpha_{n}\right)=0$, we may assume, without loss of generality, that $\rho_{n} \leq \alpha_{n}$ for all $n \geq 1$. Since $\nabla f$ is $L$ Lipschitzian, it follows that $\nabla f$ is $1 / L$-ism. By Lemma 13 (ii) we know that for $\lambda>0, \lambda \nabla f$ is $1 / \lambda L$-ism. So by Lemma 13 (iii) we deduce that $I-\lambda \nabla f$ is $\lambda L / 2$-averaged. Now since the projection $P_{C}$ is $1 / 2$-averaged, it is easy to see from Lemma 14 (iv) that the composite $P_{C}(I-\lambda \nabla f)$ is $(2+\lambda L) / 4$-averaged for $\lambda \in(0,2 / L)$. Hence, we obtain that, for each $n \geq 1$, $P_{C}\left(I-\lambda_{n} \nabla f\right)$ is $\left(2+\lambda_{n} L\right) / 4$-averaged for each $\lambda_{n} \in(0,2 / L)$. Therefore, we can write

$$
P_{C}\left(I-\lambda_{n} \nabla f\right)=\frac{2-\lambda_{n} L}{4} I+\frac{2+\lambda_{n} L}{4} T_{n}=s_{n} I+\left(1-s_{n}\right) T_{n},
$$

where $T_{n}$ is nonexpansive and $s_{n}:=s_{n}\left(\lambda_{n}\right)=\left(2-\lambda_{n} L\right) / 4 \epsilon$ $(0,1 / 2)$ for each $\lambda_{n} \in(0,2 / L)$. It is clear that

$$
\lambda_{n} \longrightarrow \frac{2}{L} \Longleftrightarrow s_{n} \longrightarrow 0 .
$$


Since $\widetilde{A}_{2}$ is $\kappa$-Lipschitz continuous, we get

$$
\left\|\widetilde{A}_{2} y_{n}-\widetilde{A}_{2} x^{*}\right\| \leq \kappa\left\|y_{n}-x^{*}\right\|, \quad \forall n \geq 1
$$

Put

$$
\begin{aligned}
\Lambda_{n}^{k}= & J_{R_{k}, \lambda_{k, n}}\left(I-\lambda_{k, n} B_{k}\right) J_{R_{k-1}, \lambda_{k-1, n}} \\
& \times\left(I-\lambda_{k-1, n} B_{k-1}\right) \cdots J_{R_{1}, \lambda_{1, n}}\left(I-\lambda_{1, n} B_{1}\right)
\end{aligned}
$$

for $k=1,2, \ldots, M$ and $\Lambda_{n}^{0}=I$, where $I$ is the identity mapping on $H$. Then, we have $v_{n}=\Lambda_{n}^{M} u_{n}$.

We divide the rest of the proof into several steps.

Step 1. We prove that $\left\{x_{n}\right\}$ is bounded.

Indeed, take $p \in \Omega$ arbitrarily. Since $p=S_{r_{n}}^{(\Theta, \varphi)}\left(p-r_{n} A p\right)$, $A$ is $\zeta$-inverse strongly monotone and $0<r_{n} \leq 2 \zeta$; utilizing the nonexpansivity of $S_{r_{n}}^{(\Theta, \varphi)}$, we have, for any $n \geq 1$,

$$
\begin{aligned}
\left\|u_{n}-p\right\|^{2}= & \left\|S_{r_{n}}^{(\Theta, \varphi)}\left(I-r_{n} A\right) x_{n}-S_{r_{n}}^{(\Theta, \varphi)}\left(I-r_{n} A\right) p\right\|^{2} \\
\leq & \left\|\left(I-r_{n} A\right) x_{n}-\left(I-r_{n} A\right) p\right\|^{2} \\
= & \left\|\left(x_{n}-p\right)-r_{n}\left(A x_{n}-A p\right)\right\|^{2} \\
= & \left\|x_{n}-p\right\|^{2}-2 r_{n}\left\langle x_{n}-p, A x_{n}-A p\right\rangle \\
& +r_{n}^{2}\left\|A x_{n}-A p\right\|^{2} \\
\leq & \left\|x_{n}-p\right\|^{2}-2 r_{n} \zeta\left\|A x_{n}-A p\right\|^{2}+r_{n}^{2}\left\|A x_{n}-A p\right\|^{2} \\
= & \left\|x_{n}-p\right\|^{2}+r_{n}\left(r_{n}-2 \zeta\right)\left\|A x_{n}-A p\right\|^{2} \\
\leq & \left\|x_{n}-p\right\|^{2} .
\end{aligned}
$$

Utilizing (21) and Lemma 20 we have

$$
\begin{aligned}
\left\|v_{n}-p\right\|= & \| J_{R_{M}, \lambda_{M, n}}\left(I-\lambda_{M, n} B_{M}\right) \Lambda_{n}^{M-1} u_{n}-J_{R_{M}, \lambda_{M, n}} \\
& \quad \times\left(I-\lambda_{M, n} B_{M}\right) \Lambda_{n}^{M-1} p \| \\
\leq & \left\|\left(I-\lambda_{M, n} B_{M}\right) \Lambda_{n}^{M-1} u_{n}-\left(I-\lambda_{M, n} B_{M}\right) \Lambda_{n}^{M-1} p\right\| \\
\leq & \left\|\Lambda_{n}^{M-1} u_{n}-\Lambda_{n}^{M-1} p\right\| \\
\vdots & \left\|\Lambda_{n}^{0} u_{n}-\Lambda_{n}^{0} p\right\|=\left\|u_{n}-p\right\| .
\end{aligned}
$$

Combining (60) and (61), we have

$$
\left\|v_{n}-p\right\| \leq\left\|x_{n}-p\right\| .
$$

Since

$$
p=P_{C}\left(I-\lambda_{n} \nabla f\right) p=s_{n} p+\left(1-s_{n}\right) T_{n} p, \quad \forall \lambda_{n} \in\left(0, \frac{2}{L}\right),
$$

where $s_{n}:=s_{n}\left(\lambda_{n}\right)=\left(2-\lambda_{n} L\right) / 4 \in(0,1 / 2)$, it is clear that $T_{n} p=p$ for each $\lambda_{n} \in(0,2 / L)$. Since $\widetilde{A}_{1}$ is $\alpha$-inverse strongly monotone and $\left\{\rho_{n}\right\}_{n=1}^{\infty} \subset(0,2 \alpha]$, utilizing the nonexpansivity of $T_{n}$, we obtain from (21), (55), and (62) that

$$
\begin{aligned}
\left\|y_{n}-p\right\| & \\
= & \left\|\beta_{n}\left(T_{n} v_{n}-p\right)+\left(1-\beta_{n}\right)\left[W_{n}\left(v_{n}-\rho_{n} \widetilde{A}_{1} v_{n}\right)-p\right]\right\| \\
\leq & \beta_{n}\left\|T_{n} v_{n}-p\right\|+\left(1-\beta_{n}\right)\left\|W_{n}\left(v_{n}-\rho_{n} \widetilde{A}_{1} v_{n}\right)-p\right\| \\
\leq & \beta_{n}\left\|v_{n}-p\right\|+\left(1-\beta_{n}\right)\left\|\left(v_{n}-\rho_{n} \widetilde{A}_{1} v_{n}\right)-p\right\| \\
= & \beta_{n}\left\|v_{n}-p\right\| \\
& +\left(1-\beta_{n}\right)\left\|\left(I-\rho_{n} \widetilde{A}_{1}\right) v_{n}-\left(I-\rho_{n} \widetilde{A}_{1}\right) p-\rho_{n} \widetilde{A}_{1} p\right\| \\
\leq & \beta_{n}\left\|v_{n}-p\right\|+\left(1-\beta_{n}\right) \\
& \times\left(\left\|\left(I-\rho_{n} \widetilde{A}_{1}\right) v_{n}-\left(I-\rho_{n} \widetilde{A}_{1}\right) p\right\|+\rho_{n}\left\|\widetilde{A}_{1} p\right\|\right) \\
\leq & \beta_{n}\left\|v_{n}-p\right\|+\left(1-\beta_{n}\right)\left(\left\|v_{n}-p\right\|+\rho_{n}\left\|\widetilde{A}_{1} p\right\|\right) \\
\leq & \left\|v_{n}-p\right\|+\rho_{n}\left\|\widetilde{A}_{1} p\right\| \leq\left\|x_{n}-p\right\|+\rho_{n}\left\|\widetilde{A}_{1} p\right\| .
\end{aligned}
$$

Utilizing Lemma 17, we obtain from (55) and $\rho_{n} \leq \alpha_{n}$ that

$$
\begin{aligned}
\left\|x_{n+1}-p\right\| & \left\|y_{n}-\mu \alpha_{n} \widetilde{A}_{2} y_{n}-p\right\| \\
\leq & \left\|\left(I-\mu \alpha_{n} \widetilde{A}_{2}\right) y_{n}-\left(I-\mu \alpha_{n} \widetilde{A}_{2}\right) p\right\| \\
& +\left\|\left(I-\mu \alpha_{n} \widetilde{A}_{2}\right) p-p\right\| \\
\leq & \left(1-\alpha_{n} \tau\right)\left\|y_{n}-p\right\|+\mu \alpha_{n}\left\|\widetilde{A}_{2} p\right\| \\
\leq & \left(1-\alpha_{n} \tau\right)\left[\left\|x_{n}-p\right\|+\rho_{n}\left\|\widetilde{A}_{1} p\right\|\right]+\alpha_{n} \mu\left\|\widetilde{A}_{2} p\right\| \\
\leq & \left(1-\alpha_{n} \tau\right)\left\|x_{n}-p\right\|+\rho_{n}\left\|\widetilde{A}_{1} p\right\|+\alpha_{n} \mu\left\|\widetilde{A}_{2} p\right\| \\
\leq & \left(1-\alpha_{n} \tau\right)\left\|x_{n}-p\right\|+\alpha_{n}\left(\left\|\widetilde{A}_{1} p\right\|+\mu\left\|\widetilde{A}_{2} p\right\|\right) \\
= & \left.\left(1-\alpha_{n} \tau\right)\left\|x_{n}-p\right\|+\alpha_{n} \tau \cdot \frac{\left\|\widetilde{A}_{1} p\right\|+\mu\left\|\widetilde{A}_{2} p\right\|}{\tau}\right\} \\
\leq & \max \left\{\left\|x_{n}-p\right\|, \frac{\left\|\widetilde{A}_{1} p\right\|+\mu\left\|\widetilde{A}_{2} p\right\|}{\tau}\right\},
\end{aligned}
$$

where $\tau:=1-\sqrt{1-\mu\left(2 \beta-\mu \kappa^{2}\right)}$. By induction, we find that

$$
\left\|x_{n}-p\right\| \leq \max \left\{\left\|x_{1}-p\right\|, \frac{\left\|\widetilde{A}_{1} p\right\|+\mu\left\|\widetilde{A}_{2} p\right\|}{\tau}\right\}, \quad \forall n \geq 1
$$

Thus, $\left\{x_{n}\right\}_{n=1}^{\infty}$ is bounded and so are the sequences $\left\{u_{n}\right\}_{n=1}^{\infty},\left\{v_{n}\right\}_{n=1}^{\infty}$ and $\left\{y_{n}\right\}_{n=1}^{\infty}$. 
Step 2. We prove that $\lim _{n \rightarrow \infty}\left(\left\|x_{n+1}-x_{n}\right\| / \rho_{n}\right)=0$.

Indeed, put $\widetilde{v}_{n}=v_{n}-\rho_{n} \widetilde{A}_{1} v_{n}$ for all $n \geq 1$. Utilizing (21) and (51), we obtain that

$$
\begin{aligned}
& \left\|v_{n+1}-v_{n}\right\| \\
& =\left\|\Lambda_{n+1}^{M} u_{n+1}-\Lambda_{n}^{M} u_{n}\right\| \\
& =\| J_{R_{M}, \lambda_{M, n+1}}\left(I-\lambda_{M, n+1} B_{M}\right) \Lambda_{n+1}^{M-1} u_{n+1} \\
& -J_{R_{M}, \lambda_{M, n}}\left(I-\lambda_{M, n} B_{M}\right) \Lambda_{n}^{M-1} u_{n} \| \\
& \leq \| J_{R_{M}, \lambda_{M, n+1}}\left(I-\lambda_{M, n+1} B_{M}\right) \Lambda_{n+1}^{M-1} u_{n+1} \\
& -J_{R_{M}, \lambda_{M, n+1}}\left(I-\lambda_{M, n} B_{M}\right) \Lambda_{n+1}^{M-1} u_{n+1} \| \\
& +\| J_{R_{M}, \lambda_{M, n+1}}\left(I-\lambda_{M, n} B_{M}\right) \Lambda_{n+1}^{M-1} u_{n+1} \\
& -J_{R_{M}, \lambda_{M, n}}\left(I-\lambda_{M, n} B_{M}\right) \Lambda_{n}^{M-1} u_{n} \| \\
& \leq\left\|\left(I-\lambda_{M, n+1} B_{M}\right) \Lambda_{n+1}^{M-1} u_{n+1}-\left(I-\lambda_{M, n} B_{M}\right) \Lambda_{n+1}^{M-1} u_{n+1}\right\| \\
& +\left\|\left(I-\lambda_{M, n} B_{M}\right) \Lambda_{n+1}^{M-1} u_{n+1}-\left(I-\lambda_{M, n} B_{M}\right) \Lambda_{n}^{M-1} u_{n}\right\| \\
& +\left|\lambda_{M, n+1}-\lambda_{M, n}\right| \\
& \times\left(\frac{1}{\lambda_{M, n+1}} \| J_{R_{M}, \lambda_{M, n+1}}\left(I-\lambda_{M, n} B_{M}\right) \Lambda_{n+1}^{M-1} u_{n+1}\right. \\
& -\left(I-\lambda_{M, n} B_{M}\right) \Lambda_{n}^{M-1} u_{n} \| \\
& +\frac{1}{\lambda_{M, n}} \|\left(I-\lambda_{M, n} B_{M}\right) \Lambda_{n+1}^{M-1} u_{n+1} \\
& \left.-J_{R_{M}, \lambda_{M, n}}\left(I-\lambda_{M, n} B_{M}\right) \Lambda_{n}^{M-1} u_{n} \|\right) \\
& \leq\left|\lambda_{M, n+1}-\lambda_{M, n}\right|\left(\left\|B_{M} \Lambda_{n+1}^{M-1} u_{n+1}\right\|+\widetilde{M}\right) \\
& +\left\|\Lambda_{n+1}^{N-1} u_{n+1}-\Lambda_{n}^{M-1} u_{n}\right\| \\
& \leq\left|\lambda_{M, n+1}-\lambda_{M, n}\right|\left(\left\|B_{M} \Lambda_{n+1}^{N-1} u_{n+1}\right\|+\widetilde{M}\right) \\
& +\left|\lambda_{M-1, n+1}-\lambda_{M-1, n}\right|\left(\left\|B_{M-1} \Lambda_{n+1}^{M-2} u_{n+1}\right\|+\widetilde{M}\right) \\
& +\left\|\Lambda_{n+1}^{M-2} u_{n+1}-\Lambda_{n}^{M-2} u_{n}\right\| \\
& \leq\left|\lambda_{M, n+1}-\lambda_{M, n}\right|\left(\left\|B_{M} \Lambda_{n+1}^{M-1} u_{n+1}\right\|+\widetilde{M}\right) \\
& +\left|\lambda_{M-1, n+1}-\lambda_{M-1, n}\right|\left(\left\|B_{M-1} \Lambda_{n+1}^{M-2} u_{n+1}\right\|+\widetilde{M}\right) \\
& +\cdots+\left|\lambda_{1, n+1}-\lambda_{1, n}\right|\left(\left\|B_{1} \Lambda_{n+1}^{0} u_{n+1}\right\|+\widetilde{M}\right) \\
& +\left\|\Lambda_{n+1}^{0} u_{n+1}-\Lambda_{n}^{0} u_{n}\right\| \\
& \leq \widetilde{M}_{0} \sum_{k=1}^{M}\left|\lambda_{k, n+1}-\lambda_{k, n}\right|+\left\|u_{n+1}-u_{n}\right\| \text {, }
\end{aligned}
$$

where

$$
\begin{gathered}
\sup _{n \geq 1}\left\{\frac{1}{\lambda_{M, n+1}} \| J_{R_{M}, \lambda_{M, n+1}}\left(I-\lambda_{M, n} B_{M}\right) \Lambda_{n+1}^{M-1} u_{n+1}\right. \\
-\left(I-\lambda_{M, n} B_{M}\right) \Lambda_{n}^{M-1} u_{n} \| \\
+\frac{1}{\lambda_{M, n}} \|\left(I-\lambda_{M, n} B_{M}\right) \Lambda_{n+1}^{M-1} u_{n+1} \\
\left.\quad-J_{R_{M}, \lambda_{M, n}}\left(I-\lambda_{M, n} B_{M}\right) \Lambda_{n}^{M-1} u_{n} \|\right\} \leq \widetilde{M},
\end{gathered}
$$

for some $\widetilde{M}>0$ and $\sup _{n \geq 1}\left\{\sum_{k=1}^{M}\left\|B_{k} \Lambda_{n+1}^{k-1} u_{n+1}\right\|+\widetilde{M}\right\} \leq \widetilde{M}_{0}$ for some $\widetilde{M}_{0}>0$. Hence, it follows from (21) and $\left\{\rho_{n}\right\}_{n=1}^{\infty} \subset$ $(0,2 \alpha]$ that

$$
\begin{aligned}
& \left\|\widetilde{v}_{n+1}-\widetilde{v}_{n}\right\| \\
& =\left\|\left(v_{n+1}-\rho_{n+1} \widetilde{A}_{1} v_{n+1}\right)-\left(v_{n}-\rho_{n} \widetilde{A}_{1} v_{n}\right)\right\| \\
& \leq\left\|\left(v_{n+1}-\rho_{n+1} \widetilde{A}_{1} v_{n+1}\right)-\left(v_{n}-\rho_{n+1} \widetilde{A}_{1} v_{n}\right)\right\| \\
& \quad+\left\|\left(v_{n}-\rho_{n+1} \widetilde{A}_{1} v_{n}\right)-\left(v_{n}-\rho_{n} \widetilde{A}_{1} v_{n}\right)\right\| \\
& \leq\left\|v_{n+1}-v_{n}\right\|+\left|\rho_{n+1}-\rho_{n}\right|\left\|\widetilde{A}_{1} v_{n}\right\| \\
& \leq \widetilde{M}_{0} \sum_{k=1}^{M}\left|\lambda_{k, n+1}-\lambda_{k, n}\right|+\left\|u_{n+1}-u_{n}\right\| \\
& \quad+\left|\rho_{n+1}-\rho_{n}\right|\left\|\widetilde{A}_{1} v_{n}\right\| .
\end{aligned}
$$

Also, utilizing (21), $\left\{r_{n}\right\}_{n=1}^{\infty} \subset[c, d] \subset(0,2 \zeta)$, and Remark 7, we deduce that

$$
\begin{aligned}
& \left\|u_{n+1}-u_{n}\right\| \\
& =\left\|S_{r_{n+1}}^{(\Theta, \varphi)}\left(I-r_{n+1} A\right) x_{n+1}-S_{r_{n}}^{(\Theta, \varphi)}\left(I-r_{n} A\right) x_{n}\right\| \\
& =\| S_{r_{n+1}}^{(\Theta, \varphi)}\left(I-r_{n+1} A\right) x_{n+1}-S_{r_{n+1}}^{(\Theta, \varphi)}\left(I-r_{n} A\right) x_{n} \\
& +S_{r_{n+1}}^{(\Theta, \varphi)}\left(I-r_{n} A\right) x_{n}-S_{r_{n}}^{(\Theta, \varphi)}\left(I-r_{n} A\right) x_{n} \| \\
& \leq\left\|S_{r_{n+1}}^{(\Theta, \varphi)}\left(I-r_{n+1} A\right) x_{n+1}-S_{r_{n+1}}^{(\Theta, \varphi)}\left(I-r_{n} A\right) x_{n}\right\| \\
& +\left\|S_{r_{n+1}}^{(\Theta, \varphi)}\left(I-r_{n} A\right) x_{n}-S_{r_{n}}^{(\Theta, \varphi)}\left(I-r_{n} A\right) x_{n}\right\| \\
& \leq\left\|\left(I-r_{n+1} A\right) x_{n+1}-\left(I-r_{n} A\right) x_{n}\right\| \\
& +\left\|S_{r_{n+1}}^{(\Theta, \varphi)}\left(I-r_{n} A\right) x_{n}-S_{r_{n}}^{(\Theta, \varphi)}\left(I-r_{n} A\right) x_{n}\right\| \\
& \leq\left\|x_{n+1}-x_{n}\right\|+\left|r_{n+1}-r_{n}\right|\left\|A x_{n}\right\| \\
& +\left\|S_{r_{n+1}}^{(\Theta, \varphi)}\left(I-r_{n} A\right) x_{n}-S_{r_{n}}^{(\Theta, \varphi)}\left(I-r_{n} A\right) x_{n}\right\| \\
& \leq\left\|x_{n+1}-x_{n}\right\|+\left|r_{n+1}-r_{n}\right|\left\|A x_{n}\right\| \\
& +\frac{\left|r_{n+1}-r_{n}\right|}{r_{n+1}} \cdot \frac{\nu}{\sigma}\left\|S_{r_{n+1}}^{(\Theta, \varphi)}\left(I-r_{n} A\right) x_{n}-\left(I-r_{n} A\right) x_{n}\right\|
\end{aligned}
$$




$$
\begin{aligned}
& \leq\left\|x_{n+1}-x_{n}\right\|+\left|r_{n+1}-r_{n}\right| \\
& \quad \times\left(\left\|A x_{n}\right\|+\frac{v}{c \sigma}\left\|S_{r_{n+1}}^{(\Theta, \varphi)}\left(I-r_{n} A\right) x_{n}-\left(I-r_{n} A\right) x_{n}\right\|\right) \\
& \leq\left\|x_{n+1}-x_{n}\right\|+\left|r_{n+1}-r_{n}\right| \widetilde{M}_{1},
\end{aligned}
$$

where $\sup _{n \geq 1}\left\{\left\|A x_{n}\right\|+(\nu / c \sigma) \| S_{r_{n+1}}^{(\Theta, \varphi)}\left(I-r_{n} A\right) x_{n}-(I-\right.$ $\left.\left.r_{n} A\right) x_{n} \|\right\} \leq \widetilde{M}_{1}$ for some $\widetilde{M}_{1}>0$.

In the meantime, from (31), since $S_{i}$ and $U_{i, n}$ for $i=$ $1,2, \ldots, N$ are nonexpansive, we get

$$
\begin{aligned}
& \left\|W_{n+1} \widetilde{v}_{n}-W_{n} \widetilde{v}_{n}\right\| \\
& =\| \mu_{N, n+1} S_{N} U_{N-1, n+1} \widetilde{v}_{n}+\left(1-\mu_{N, n+1}\right) \widetilde{v}_{n} \\
& \quad-\mu_{N, n} S_{N} U_{N-1, n} \widetilde{v}_{n}-\left(1-\mu_{N, n}\right) \widetilde{v}_{n} \| \\
& \leq\left|\mu_{N, n+1}-\mu_{N, n}\right|\left\|\widetilde{v}_{n}\right\| \\
& \quad+\left\|\mu_{N, n+1} S_{N} U_{N-1, n+1} \widetilde{v}_{n}-\mu_{N, n} S_{N} U_{N-1, n} \widetilde{v}_{n}\right\| \\
& \leq\left|\mu_{N, n+1}-\mu_{N, n}\right|\left\|\widetilde{v}_{n}\right\| \\
& \quad+\left\|\mu_{N, n+1}\left(S_{N} U_{N-1, n+1} \widetilde{v}_{n}-S_{N} U_{N-1, n} \widetilde{v}_{n}\right)\right\| \\
& \quad+\left|\mu_{N, n+1}-\mu_{N, n}\right|\left\|S_{N} U_{N-1, n} \widetilde{v}_{n}\right\| \\
& \leq \widetilde{M}_{2}\left|\mu_{N, n+1}-\mu_{N, n}\right| \\
& \quad+\mu_{N, n+1}\left\|U_{N-1, n+1} \widetilde{v}_{n}-U_{N-1, n} \widetilde{v}_{n}\right\|,
\end{aligned}
$$

and by (31),

$$
\begin{aligned}
\left\|U_{N-1, n+1} \tilde{v}_{n}-U_{N-1, n} \tilde{v}_{n}\right\| \\
=\| \mu_{N-1, n+1} S_{N-1} U_{N-2, n+1} \widetilde{v}_{n}+\left(1-\mu_{N-1, n+1}\right) \widetilde{v}_{n} \\
\quad \quad-\mu_{N-1, n} S_{N-1} U_{N-2, n} \widetilde{v}_{n}-\left(1-\mu_{N-1, n}\right) \widetilde{v}_{n} \| \\
\leq\left|\mu_{N-1, n+1}-\mu_{N-1, n}\right|\left\|\widetilde{v}_{n}\right\| \\
\quad+\left\|\mu_{N-1, n+1} S_{N-1} U_{N-2, n+1} \widetilde{v}_{n}-\mu_{N-1, n} S_{N-1} U_{N-2, n} \widetilde{v}_{n}\right\| \\
\leq\left|\mu_{N-1, n+1}-\mu_{N-1, n}\right|\left\|\widetilde{v}_{n}\right\| \\
\quad+\mu_{N-1, n+1}\left\|S_{N-1} U_{N-2, n+1} \widetilde{v}_{n}-S_{N-1} U_{N-2, n} \widetilde{v}_{n}\right\| \\
\quad+\left|\mu_{N-1, n+1}-\mu_{N-1, n}\right|\left\|S_{N-1} U_{N-2, n} \widetilde{v}_{n}\right\| \\
\leq \widetilde{M}_{2}\left|\mu_{N-1, n+1}-\mu_{N-1, n}\right| \\
\quad+\mu_{N-1, n+1}\left\|U_{N-2, n+1} \widetilde{v}_{n}-U_{N-2, n} \widetilde{v}_{n}\right\| \\
\leq \widetilde{M}_{2}\left|\mu_{N-1, n+1}-\mu_{N-1, n}\right|+\left\|U_{N-2, n+1} \widetilde{v}_{n}-U_{N-2, n} \widetilde{v}_{n}\right\|,
\end{aligned}
$$

where $\sup _{n \geq 1}\left\{\left\|\widetilde{v}_{n}\right\|+\sum_{i=1}^{N}\left\|S_{i} U_{i-1, n} \widetilde{v}_{n}\right\|\right\} \leq \widetilde{M}_{2}, \quad i=1,2, \ldots, N$ for some $\widetilde{M}_{2}>0$. Therefore, we have

$$
\begin{aligned}
\left\|U_{N-1, n+1} \widetilde{v}_{n}-U_{N-1, n} \widetilde{v}_{n}\right\| & \\
\leq & \widetilde{M}_{2}\left|\mu_{N-1, n+1}-\mu_{N-1, n}\right| \\
& +\left\|U_{N-2, n+1} \widetilde{v}_{n}-U_{N-2, n} \widetilde{v}_{n}\right\| \\
\leq & \widetilde{M}_{2}\left|\mu_{N-1, n+1}-\mu_{N-1, n}\right|+\widetilde{M}_{2}\left|\mu_{N-2, n+1}-\mu_{N-2, n}\right| \\
& +\left\|U_{N-3, n+1} \widetilde{v}_{n}-U_{N-3, n} \widetilde{v}_{n}\right\| \\
& \quad \vdots \\
\leq & \widetilde{M}_{2} \sum_{i=2}^{N-1}\left|\mu_{i, n+1}-\mu_{i, n}\right|+\left\|U_{1, n+1} \widetilde{v}_{n}-U_{1, n} \widetilde{v}_{n}\right\| \\
= & \left\|\mu_{1, n+1} S_{1} \widetilde{v}_{n}+\left(1-\mu_{1, n+1}\right) \widetilde{v}_{n}-\mu_{1, n} S_{1} \widetilde{v}_{n}-\left(1-\mu_{1, n}\right) \widetilde{v}_{n}\right\| \\
& +\widetilde{M}_{2} \sum_{i=2}^{N-1}\left|\mu_{i, n+1}-\mu_{i, n}\right|,
\end{aligned}
$$

and hence

$$
\begin{aligned}
& \left\|U_{N-1, n+1} \widetilde{v}_{n}-U_{N-1, n} \widetilde{v}_{n}\right\| \\
& \leq\left|\mu_{1, n+1}-\mu_{1, n}\right|\left\|\widetilde{v}_{n}\right\|+\left\|\mu_{1, n+1} S_{1} \widetilde{v}_{n}-\mu_{1, n} S_{1} \widetilde{v}_{n}\right\| \\
& \quad+\widetilde{M}_{2} \sum_{i=2}^{N-1}\left|\mu_{i, n+1}-\mu_{i, n}\right| \leq \widetilde{M}_{2} \sum_{i=1}^{N-1}\left|\mu_{i, n+1}-\mu_{i, n}\right|
\end{aligned}
$$

which immediately yields

$$
\begin{aligned}
& \left\|W_{n+1} \widetilde{v}_{n+1}-W_{n} \widetilde{v}_{n}\right\| \\
& \leq\left\|W_{n+1} \widetilde{v}_{n+1}-W_{n+1} \widetilde{v}_{n}\right\|+\left\|W_{n+1} \widetilde{v}_{n}-W_{n} \widetilde{v}_{n}\right\| \\
& \leq\left\|\widetilde{v}_{n+1}-\widetilde{v}_{n}\right\|+\widetilde{M}_{2}\left|\mu_{N, n+1}-\mu_{N, n}\right| \\
& \quad+\mu_{N, n+1}\left\|U_{N-1, n+1} \widetilde{v}_{n}-U_{N-1, n} \widetilde{v}_{n}\right\| \\
& \leq\left\|\widetilde{v}_{n+1}-\widetilde{v}_{n}\right\|+\widetilde{M}_{2}\left|\mu_{N, n+1}-\mu_{N, n}\right| \\
& \quad+\mu_{N, n+1} \widetilde{M}_{2} \sum_{i=1}^{N-1}\left|\mu_{i, n+1}-\mu_{i, n}\right| \\
& \leq\left\|\widetilde{v}_{n+1}-\widetilde{v}_{n}\right\|+\widetilde{M}_{2} \sum_{i=1}^{N}\left|\mu_{i, n+1}-\mu_{i, n}\right| .
\end{aligned}
$$

Furthermore, since $\nabla f$ is $1 / L$-ism, $P_{C}\left(I-\lambda_{n} \nabla f\right)$ is nonexpansive for $\lambda_{n} \in(0,2 / L)$. So, it follows that, for any given $p \in \Omega$,

$$
\begin{aligned}
& \left\|P_{C}\left(I-\lambda_{n+1} \nabla f\right) v_{n}\right\| \\
& \quad \leq\left\|P_{C}\left(I-\lambda_{n+1} \nabla f\right) v_{n}-p\right\|+\|p\| \\
& \quad=\left\|P_{C}\left(I-\lambda_{n+1} \nabla f\right) v_{n}-P_{C}\left(I-\lambda_{n+1} \nabla f\right) p\right\|+\|p\|
\end{aligned}
$$




$$
\begin{aligned}
& \leq\left\|v_{n}-p\right\|+\|p\| \\
& \leq\left\|v_{n}\right\|+2\|p\| .
\end{aligned}
$$

This together with the boundedness of $\left\{v_{n}\right\}$ implies that $\left\{P_{C}\left(I-\lambda_{n+1} \nabla f\right) v_{n}\right\}$ is bounded. Also, observe that

$$
\begin{aligned}
& \left\|T_{n+1} v_{n}-T_{n} v_{n}\right\| \\
& =\| \frac{4 P_{C}\left(I-\lambda_{n+1} \nabla f\right)-\left(2-\lambda_{n+1} L\right) I}{2+\lambda_{n+1} L} v_{n} \\
& -\frac{4 P_{C}\left(I-\lambda_{n} \nabla f\right)-\left(2-\lambda_{n} L\right) I}{2+\lambda_{n} L} v_{n} \| \\
& \leq\left\|\frac{4 P_{C}\left(I-\lambda_{n+1} \nabla f\right)}{2+\lambda_{n+1} L} v_{n}-\frac{4 P_{C}\left(I-\lambda_{n} \nabla f\right)}{2+\lambda_{n} L} v_{n}\right\| \\
& +\left\|\frac{2-\lambda_{n} L}{2+\lambda_{n} L} v_{n}-\frac{2-\lambda_{n+1} L}{2+\lambda_{n+1} L} v_{n}\right\| \\
& =\|\left(4\left(2+\lambda_{n} L\right) P_{C}\left(I-\lambda_{n+1} \nabla f\right) v_{n}\right. \\
& \left.-4\left(2+\lambda_{n+1} L\right) P_{C}\left(I-\lambda_{n} \nabla f\right) v_{n}\right) \\
& \times\left(\left(2+\lambda_{n+1} L\right)\left(2+\lambda_{n} L\right)\right)^{-1} \| \\
& +\frac{4 L\left|\lambda_{n+1}-\lambda_{n}\right|}{\left(2+\lambda_{n+1} L\right)\left(2+\lambda_{n} L\right)}\left\|v_{n}\right\| \\
& =\|\left(4 L\left(\lambda_{n}-\lambda_{n+1}\right) P_{C}\left(I-\lambda_{n+1} \nabla f\right) v_{n}+4\left(2+\lambda_{n+1} L\right)\right. \\
& \left.\times\left(P_{C}\left(I-\lambda_{n+1} \nabla f\right) v_{n}-P_{C}\left(I-\lambda_{n} \nabla f\right) v_{n}\right)\right) \\
& \times\left(\left(2+\lambda_{n+1} L\right)\left(2+\lambda_{n} L\right)\right)^{-1} \| \\
& +\frac{4 L\left|\lambda_{n+1}-\lambda_{n}\right|}{\left(2+\lambda_{n+1} L\right)\left(2+\lambda_{n} L\right)}\left\|v_{n}\right\| \\
& \leq \frac{4 L\left|\lambda_{n}-\lambda_{n+1}\right|\left\|P_{C}\left(I-\lambda_{n+1} \nabla f\right) v_{n}\right\|}{\left(2+\lambda_{n+1} L\right)\left(2+\lambda_{n} L\right)} \\
& +\frac{4\left(2+\lambda_{n+1} L\right)\left\|P_{C}\left(I-\lambda_{n+1} \nabla f\right) v_{n}-P_{C}\left(I-\lambda_{n} \nabla f\right) v_{n}\right\|}{\left(2+\lambda_{n+1} L\right)\left(2+\lambda_{n} L\right)} \\
& +\frac{4 L\left|\lambda_{n+1}-\lambda_{n}\right|}{\left(2+\lambda_{n+1} L\right)\left(2+\lambda_{n} L\right)}\left\|v_{n}\right\| \\
& \leq\left|\lambda_{n+1}-\lambda_{n}\right| \\
& \times\left[L\left\|P_{C}\left(I-\lambda_{n+1} \nabla f\right) v_{n}\right\|+4\left\|\nabla f\left(v_{n}\right)\right\|+L\left\|v_{n}\right\|\right] \\
& \leq \widetilde{M}_{3}\left|\lambda_{n+1}-\lambda_{n}\right| \text {, }
\end{aligned}
$$

where $\sup _{n \geq 1}\left\{L\left\|P_{C}\left(I-\lambda_{n+1} \nabla f\right) v_{n}\right\|+4\left\|\nabla f\left(v_{n}\right)\right\|+L\left\|v_{n}\right\|\right\} \leq \widetilde{M}_{3}$ for some $\widetilde{M}_{3}>0$. So, we conclude that

$$
\begin{aligned}
& \left\|T_{n+1} v_{n+1}-T_{n} v_{n}\right\| \\
& \quad \leq\left\|T_{n+1} v_{n+1}-T_{n+1} v_{n}\right\|+\left\|T_{n+1} v_{n}-T_{n} v_{n}\right\| \\
& \quad \leq\left\|v_{n+1}-v_{n}\right\|+\widetilde{M}_{3}\left|\lambda_{n+1}-\lambda_{n}\right| \\
& \quad \leq\left\|v_{n+1}-v_{n}\right\|+\frac{4 \widetilde{M}_{3}}{L}\left|s_{n+1}-s_{n}\right| .
\end{aligned}
$$

Now, simple calculation shows that

$$
\begin{aligned}
y_{n+1}-y_{n}= & \beta_{n}\left(T_{n+1} v_{n+1}-T_{n} v_{n}\right) \\
& +\left(\beta_{n+1}-\beta_{n}\right)\left(T_{n+1} v_{n+1}-W_{n+1} \widetilde{v}_{n+1}\right) \\
& +\left(1-\beta_{n}\right)\left(W_{n+1} \widetilde{v}_{n+1}-W_{n} \widetilde{v}_{n}\right) .
\end{aligned}
$$

So, utilizing (67)-(78), from $\left\{\alpha_{n}\right\} \subset(0,1],\left\{\rho_{n}\right\} \subset(0,2 \alpha]$ and $\mu \in\left(0,2 \beta / \kappa^{2}\right)$, we deduce that

$$
\begin{aligned}
& \left\|y_{n+1}-y_{n}\right\| \\
& \leq \beta_{n}\left\|T_{n+1} v_{n+1}-T_{n} v_{n}\right\| \\
& +\left|\beta_{n+1}-\beta_{n}\right|\left\|T_{n+1} v_{n+1}-W_{n+1} \widetilde{v}_{n+1}\right\| \\
& +\left(1-\beta_{n}\right)\left\|W_{n+1} \widetilde{v}_{n+1}-W_{n} \widetilde{v}_{n}\right\| \\
& \leq \beta_{n}\left[\left\|v_{n+1}-v_{n}\right\|+\frac{4 \widetilde{M}_{3}}{L}\left|s_{n+1}-s_{n}\right|\right] \\
& +\left|\beta_{n+1}-\beta_{n}\right|\left\|T_{n+1} v_{n+1}-W_{n+1} \widetilde{v}_{n+1}\right\| \\
& +\left(1-\beta_{n}\right)\left[\left\|\widetilde{v}_{n+1}-\widetilde{v}_{n}\right\|+\widetilde{M}_{2} \sum_{i=1}^{N}\left|\mu_{i, n+1}-\mu_{i, n}\right|\right] \\
& \leq \beta_{n}\left[\widetilde{M}_{0} \sum_{k=1}^{M}\left|\lambda_{k, n+1}-\lambda_{k, n}\right|\right. \\
& \left.+\left\|u_{n+1}-u_{n}\right\|+\frac{4 \widetilde{M}_{3}}{L}\left|s_{n+1}-s_{n}\right|\right] \\
& +\left|\beta_{n+1}-\beta_{n}\right|\left\|T_{n+1} v_{n+1}-W_{n+1} \widetilde{v}_{n+1}\right\|+\left(1-\beta_{n}\right) \\
& \times\left[\widetilde{M}_{0} \sum_{k=1}^{M}\left|\lambda_{k, n+1}-\lambda_{k, n}\right|+\left\|u_{n+1}-u_{n}\right\|\right. \\
& \left.+\left|\rho_{n+1}-\rho_{n}\right|\left\|\widetilde{A}_{1} v_{n}\right\|+\widetilde{M}_{2} \sum_{i=1}^{N}\left|\mu_{i, n+1}-\mu_{i, n}\right|\right] \\
& \leq\left\|u_{n+1}-u_{n}\right\|+\widetilde{M}_{0} \sum_{k=1}^{M}\left|\lambda_{k, n+1}-\lambda_{k, n}\right| \\
& +\frac{4 \widetilde{M}_{3}}{L}\left|s_{n+1}-s_{n}\right|+\left|\beta_{n+1}-\beta_{n}\right| \| T_{n+1} v_{n+1}-W_{n+1} \widetilde{v}_{n+1}|| \\
& +\left|\rho_{n+1}-\rho_{n}\right||| \widetilde{A}_{1} v_{n}||+\widetilde{M}_{2} \sum_{i=1}^{N}\left|\mu_{i, n+1}-\mu_{i, n}\right|
\end{aligned}
$$




$$
\begin{aligned}
& \leq|| x_{n+1}-x_{n} \|+\left|r_{n+1}-r_{n}\right| \widetilde{M}_{1} \\
& \quad+\widetilde{M}_{0} \sum_{k=1}^{M}\left|\lambda_{k, n+1}-\lambda_{k, n}\right|+\frac{4 \widetilde{M}_{3}}{L}\left|s_{n+1}-s_{n}\right| \\
& \quad+\left|\beta_{n+1}-\beta_{n}\right| \| T_{n+1} v_{n+1}-W_{n+1} \widetilde{v}_{n+1}|| \\
& \quad+\left|\rho_{n+1}-\rho_{n}\right|\left\|\widetilde{A}_{1} v_{n}\right\|+\widetilde{M}_{2} \sum_{i=1}^{N}\left|\mu_{i, n+1}-\mu_{i, n}\right|,
\end{aligned}
$$

and by Lemma 17,

$$
\begin{aligned}
& \left\|x_{n+2}-x_{n+1}\right\| \\
& =\left\|\left(I-\mu \alpha_{n+1} \widetilde{A}_{2}\right) y_{n+1}-\left(I-\mu \alpha_{n} \widetilde{A}_{2}\right) y_{n}\right\| \\
& \leq\left\|\left(I-\mu \alpha_{n+1} \widetilde{A}_{2}\right) y_{n+1}-\left(I-\mu \alpha_{n+1} \widetilde{A}_{2}\right) y_{n}\right\| \\
& +\left\|\left(I-\mu \alpha_{n+1} \widetilde{A}_{2}\right) y_{n}-\left(I-\mu \alpha_{n} \widetilde{A}_{2}\right) y_{n}\right\| \\
& \leq\left(1-\alpha_{n+1} \tau\right)\left\|y_{n+1}-y_{n}\right\|+\left|\alpha_{n+1}-\alpha_{n}\right| \mu\left\|\widetilde{A}_{2} y_{n}\right\| \\
& \leq\left(1-\alpha_{n+1} \tau\right) \\
& \times\left[\left\|x_{n+1}-x_{n}\right\|+\left|r_{n+1}-r_{n}\right| \widetilde{M}_{1}\right. \\
& +\widetilde{M}_{0} \sum_{k=1}^{M}\left|\lambda_{k, n+1}-\lambda_{k, n}\right|+\frac{4 \widetilde{M}_{3}}{L}\left|s_{n+1}-s_{n}\right| \\
& +\left|\beta_{n+1}-\beta_{n}\right|\left\|T_{n+1} v_{n+1}-W_{n+1} \widetilde{v}_{n+1}\right\| \\
& \left.+\left|\rho_{n+1}-\rho_{n}\right|\left\|\widetilde{A}_{1} v_{n}\right\|+\widetilde{M}_{2} \sum_{i=1}^{N}\left|\mu_{i, n+1}-\mu_{i, n}\right|\right] \\
& +\left|\alpha_{n+1}-\alpha_{n}\right| \mu\left\|\widetilde{A}_{2} y_{n}\right\| \\
& \leq\left(1-\alpha_{n+1} \tau\right)\left\|x_{n+1}-x_{n}\right\| \\
& +\left|r_{n+1}-r_{n}\right| \widetilde{M}_{1}+\widetilde{M}_{0} \sum_{k=1}^{M}\left|\lambda_{k, n+1}-\lambda_{k, n}\right| \\
& +\frac{4 \widetilde{M}_{3}}{L}\left|s_{n+1}-s_{n}\right| \\
& +\left|\beta_{n+1}-\beta_{n}\right|\left\|T_{n+1} v_{n+1}-W_{n+1} \widetilde{v}_{n+1}\right\| \\
& +\left|\rho_{n+1}-\rho_{n}\right|\left\|\widetilde{A}_{1} v_{n}\right\|+\widetilde{M}_{2} \sum_{i=1}^{N}\left|\mu_{i, n+1}-\mu_{i, n}\right| \\
& +\left|\alpha_{n+1}-\alpha_{n}\right| \mu\left\|\widetilde{A}_{2} y_{n}\right\| \\
& \leq\left(1-\alpha_{n+1} \tau\right)\left\|x_{n+1}-x_{n}\right\|+\left|r_{n+1}-r_{n}\right| \widetilde{M}_{4} \\
& +\widetilde{M}_{4} \sum_{k=1}^{M}\left|\lambda_{k, n+1}-\lambda_{k, n}\right|+\widetilde{M}_{4}\left|s_{n+1}-s_{n}\right|
\end{aligned}
$$

$$
\begin{gathered}
+\left|\beta_{n+1}-\beta_{n}\right| \widetilde{M}_{4}+\left|\rho_{n+1}-\rho_{n}\right| \widetilde{M}_{4} \\
+\widetilde{M}_{4} \sum_{i=1}^{N}\left|\mu_{i, n+1}-\mu_{i, n}\right|+\left|\alpha_{n+1}-\alpha_{n}\right| \widetilde{M}_{4} \\
=\left(1-\alpha_{n+1} \tau\right)\left\|x_{n+1}-x_{n}\right\| \\
+\widetilde{M}_{4}\left(\sum_{k=1}^{M}\left|\lambda_{k, n+1}-\lambda_{k, n}\right|+\sum_{i=1}^{N}\left|\mu_{i, n+1}-\mu_{i, n}\right|\right. \\
\quad+\left|r_{n+1}-r_{n}\right|+\left|s_{n+1}-s_{n}\right|+\left|\alpha_{n+1}-\alpha_{n}\right| \\
\left.\quad+\left|\beta_{n+1}-\beta_{n}\right|+\left|\rho_{n+1}-\rho_{n}\right|\right),
\end{gathered}
$$

where $\sup _{n \geq 1}\left\{\widetilde{M}_{0}+\widetilde{M}_{1}+\widetilde{M}_{2}+\left(4 \widetilde{M}_{3} / L\right)+\left\|T_{n} v_{n}-W_{n} \widetilde{v}_{n}\right\|+\right.$ $\left.\left\|\widetilde{A}_{1} v_{n}\right\|+\mu\left\|\widetilde{A}_{2} y_{n}\right\|\right\} \leq \widetilde{M}_{4}$ for some $\widetilde{M}_{4}>0$. Consequently,

$$
\begin{aligned}
& \frac{\left\|x_{n+1}-x_{n}\right\|}{\rho_{n}} \\
& \leq\left(1-\alpha_{n} \tau\right) \frac{\left\|x_{n}-x_{n-1}\right\|}{\rho_{n}} \\
& +\widetilde{M}_{4}\left(\sum_{k=1}^{M} \frac{\left|\lambda_{k, n}-\lambda_{k, n-1}\right|}{\rho_{n}}+\sum_{i=1}^{N} \frac{\left|\mu_{i, n}-\mu_{i, n-1}\right|}{\rho_{n}}\right. \\
& +\frac{\left|r_{n}-r_{n-1}\right|}{\rho_{n}}+\frac{\left|s_{n}-s_{n-1}\right|}{\rho_{n}}+\frac{\left|\alpha_{n}-\alpha_{n-1}\right|}{\rho_{n}} \\
& \left.+\left(1-\tau \alpha_{n}\right) \frac{|| x_{n}-x_{n-1} \|}{\rho_{n-1}}+\frac{\left|\rho_{n}-\rho_{n-1}\right|}{\rho_{n}}\right) \\
& +\left(1-\tau \alpha_{n}\right)|| \mid x_{n}-x_{n-1} \|\left(\frac{1}{\rho_{n}}-\frac{1}{\rho_{n-1}}\right) \\
& +\widetilde{M}_{4}\left(\sum_{k=1}^{M} \frac{\left|\lambda_{k, n}-\lambda_{k, n-1}\right|}{\rho_{n}}+\sum_{i=1}^{N} \frac{\left|\mu_{i, n}-\mu_{i, n-1}\right|}{\rho_{n}}\right. \\
& +\tau \alpha_{n} \cdot \frac{\widetilde{M}_{5}}{\tau}\left\{\frac{1}{\alpha_{n}}\left|\frac{1}{\rho_{n}}-\frac{1}{\rho_{n-1}}\right|\right. \\
& +\left(1-\tau \alpha_{n}\right) \frac{|| x_{n}-x_{n-1} \mid}{\rho_{n}}+\frac{\left|s_{n}-s_{n-1}\right|}{\rho_{n}}+\frac{\left|\alpha_{n}-\alpha_{n-1}\right|}{\rho_{n}} \\
& \left.+\beta_{n-1} \mid \frac{\left|\beta_{n}-\rho_{n-1}\right|}{\rho_{n}}\right)
\end{aligned}
$$




$$
\begin{aligned}
& +\frac{\left|r_{n}-r_{n-1}\right|}{\alpha_{n} \rho_{n}}+\frac{\left|s_{n}-s_{n-1}\right|}{\alpha_{n} \rho_{n}}+\frac{\left|\beta_{n}-\beta_{n-1}\right|}{\alpha_{n} \rho_{n}} \\
& \left.+\frac{1}{\rho_{n}}\left|1-\frac{\alpha_{n-1}}{\alpha_{n}}\right|+\frac{1}{\alpha_{n}}\left|1-\frac{\rho_{n-1}}{\rho_{n}}\right|\right\},
\end{aligned}
$$

where $\sup _{n \geq 1}\left\{\left\|x_{n+1}-x_{n}\right\|+\widetilde{M}_{4}\right\} \leq \widetilde{M}_{5}$ for some $\widetilde{M}_{5}>0$. From $\left|s_{n}-s_{n-1}\right| / \alpha_{n} \rho_{n} \rightarrow 0$ and (iii)-(vi) it follows that $\sum_{n=1}^{\infty} \tau \alpha_{n}=$ $\infty$ and

$$
\begin{aligned}
\lim _{n \rightarrow \infty} \frac{\widetilde{M}_{5}}{\tau}\left\{\frac{1}{\alpha_{n}}\left|\frac{1}{\rho_{n}}-\frac{1}{\rho_{n-1}}\right|+\sum_{k=1}^{M} \frac{\left|\lambda_{k, n}-\lambda_{k, n-1}\right|}{\alpha_{n} \rho_{n}}\right. \\
+\sum_{i=1}^{N} \frac{\left|\mu_{i, n}-\mu_{i, n-1}\right|}{\alpha_{n} \rho_{n}}+\frac{\left|r_{n}-r_{n-1}\right|}{\alpha_{n} \rho_{n}} \\
+\frac{\left|s_{n}-s_{n-1}\right|}{\alpha_{n} \rho_{n}}+\frac{\left|\beta_{n}-\beta_{n-1}\right|}{\alpha_{n} \rho_{n}} \\
\left.+\frac{1}{\rho_{n}}\left|1-\frac{\alpha_{n-1}}{\alpha_{n}}\right|+\frac{1}{\alpha_{n}}\left|1-\frac{\rho_{n-1}}{\rho_{n}}\right|\right\}=0 .
\end{aligned}
$$

Thus, utilizing Lemma 18, we immediately conclude that

$$
\lim _{n \rightarrow \infty} \frac{\left\|x_{n+1}-x_{n}\right\|}{\rho_{n}}=0
$$

So, from $\rho_{n} \rightarrow 0$ it follows that

$$
\lim _{n \rightarrow \infty}\left\|x_{n+1}-x_{n}\right\|=0
$$

Step 3. We prove that $\lim _{n \rightarrow \infty}\left\|x_{n}-u_{n}\right\|=0, \lim _{n \rightarrow \infty} \| x_{n}-$ $v_{n}\left\|=0, \lim _{n \rightarrow \infty}\right\| v_{n}-P_{C}(I-(2 / L) \nabla f) v_{n} \|=0$, and $\lim _{n \rightarrow \infty}\left\|\widetilde{v}_{n}-W \widetilde{v}_{n}\right\|=0$, where $\widetilde{v}_{n}=v_{n}-\rho_{n} \widetilde{A}_{1} v_{n}$.

Indeed, utilizing Lemmas 10 and 11 (b), from (55) and (62), we get

$$
\begin{aligned}
\left\|x_{n+1}-p\right\|^{2} & \\
= & \left\|\left(I-\mu \alpha_{n} \widetilde{A}_{2}\right) y_{n}-p\right\|^{2} \\
= & \|\left(I-\mu \alpha_{n} \widetilde{A}_{2}\right) y_{n}-\left(I-\mu \alpha_{n} \widetilde{A}_{2}\right) p \\
& \quad+\left(I-\mu \alpha_{n} \widetilde{A}_{2}\right) p-p \|^{2} \\
\leq & \left\|\left(I-\mu \alpha_{n} \widetilde{A}_{2}\right) y_{n}-\left(I-\mu \alpha_{n} \widetilde{A}_{2}\right) p\right\|^{2} \\
& -2 \mu \alpha_{n}\left\langle\widetilde{A}_{2} p, x_{n+1}-p\right\rangle \\
\leq & \left(1-\alpha_{n} \tau\right)\left\|y_{n}-p\right\|^{2}-2 \mu \alpha_{n}\left\langle\widetilde{A}_{2} p, x_{n+1}-p\right\rangle \\
\leq & \left\|y_{n}-p\right\|^{2}-2 \mu \alpha_{n}\left\langle\widetilde{A}_{2} p, x_{n+1}-p\right\rangle
\end{aligned}
$$

$$
\begin{aligned}
& =\left\|\beta_{n}\left(T_{n} v_{n}-p\right)+\left(1-\beta_{n}\right)\left(W_{n} \widetilde{v}_{n}-p\right)\right\|^{2} \\
& -2 \mu \alpha_{n}\left\langle\widetilde{A}_{2} p, x_{n+1}-p\right\rangle \\
& =\beta_{n}\left\|T_{n} v_{n}-p\right\|^{2}+\left(1-\beta_{n}\right)\left\|W_{n} \widetilde{v}_{n}-p\right\|^{2} \\
& -\beta_{n}\left(1-\beta_{n}\right)\left\|T_{n} v_{n}-W_{n} \widetilde{v}_{n}\right\|^{2}-2 \mu \alpha_{n}\left\langle\widetilde{A}_{2} p, x_{n+1}-p\right\rangle \\
& \leq \beta_{n}\left\|T_{n} v_{n}-p\right\|^{2}+\left(1-\beta_{n}\right)\left\|\widetilde{v}_{n}-p\right\|^{2} \\
& -\beta_{n}\left(1-\beta_{n}\right)\left\|T_{n} v_{n}-W_{n} \widetilde{v}_{n}\right\|^{2} \\
& -2 \mu \alpha_{n}\left\langle\widetilde{A}_{2} p, x_{n+1}-p\right\rangle \\
& =\beta_{n}\left\|T_{n} v_{n}-p\right\|^{2}+\left(1-\beta_{n}\right)\left\|v_{n}-p-\rho_{n} \widetilde{A}_{1} v_{n}\right\|^{2} \\
& -\beta_{n}\left(1-\beta_{n}\right)\left\|T_{n} v_{n}-W_{n} \widetilde{v}_{n}\right\|^{2}-2 \mu \alpha_{n}\left\langle\widetilde{A}_{2} p, x_{n+1}-p\right\rangle \\
& \leq \beta_{n}\left\|T_{n} v_{n}-p\right\|^{2} \\
& +\left(1-\beta_{n}\right)\left(\left\|v_{n}-p\right\|^{2}-2 \rho_{n}\left\langle\widetilde{A}_{1} v_{n}, \widetilde{v}_{n}-p\right\rangle\right) \\
& -\beta_{n}\left(1-\beta_{n}\right)\left\|T_{n} v_{n}-W_{n} \widetilde{v}_{n}\right\|^{2}-2 \mu \alpha_{n}\left\langle\widetilde{A}_{2} p, x_{n+1}-p\right\rangle \\
& \leq\left\|v_{n}-p\right\|^{2}-2 \rho_{n}\left(1-\beta_{n}\right)\left\langle\widetilde{A}_{1} v_{n}, \widetilde{v}_{n}-p\right\rangle \\
& -\beta_{n}\left(1-\beta_{n}\right)\left\|T_{n} v_{n}-W_{n} \widetilde{v}_{n}\right\|^{2}-2 \mu \alpha_{n}\left\langle\widetilde{A}_{2} p, x_{n+1}-p\right\rangle \\
& \leq\left\|x_{n}-p\right\|^{2}-2 \rho_{n}\left(1-\beta_{n}\right)\left\langle\widetilde{A}_{1} v_{n}, \widetilde{v}_{n}-p\right\rangle \\
& -\beta_{n}\left(1-\beta_{n}\right)\left\|T_{n} v_{n}-W_{n} \widetilde{v}_{n}\right\|^{2} \\
& -2 \mu \alpha_{n}\left\langle\widetilde{A}_{2} p, x_{n+1}-p\right\rangle,
\end{aligned}
$$

which implies that

$$
\begin{aligned}
& \beta_{n}\left(1-\beta_{n}\right)\left\|T_{n} v_{n}-W_{n} \widetilde{v}_{n}\right\|^{2} \\
& \leq\left\|x_{n}-p\right\|^{2}-\left\|x_{n+1}-p\right\|^{2} \\
& \quad-2 \rho_{n}\left(1-\beta_{n}\right)\left\langle\widetilde{A}_{1} v_{n}, \widetilde{v}_{n}-p\right\rangle-2 \mu \alpha_{n}\left\langle\widetilde{A}_{2} p, x_{n+1}-p\right\rangle \\
& \leq\left\|x_{n}-x_{n+1}\right\|\left(\left\|x_{n}-p\right\|+\left\|x_{n+1}-p\right\|\right) \\
&+2 \rho_{n}\left\|\widetilde{A}_{1} v_{n}\right\|\left\|\widetilde{v}_{n}-p\right\|+2 \mu \alpha_{n}\left\|\widetilde{A}_{2} p\right\|\left\|x_{n+1}-p\right\| .
\end{aligned}
$$

Since $\alpha_{n} \rightarrow 0, \rho_{n} \rightarrow 0,\left\|x_{n+1}-x_{n}\right\| \rightarrow 0$ and $\left\{x_{n}\right\},\left\{v_{n}\right\},\left\{\widetilde{v}_{n}\right\}$ are bounded sequences, it follows from $\left\{\beta_{n}\right\}_{n=1}^{\infty} \subset[a, b] \subset$ $(0,1)$ that

$$
\lim _{n \rightarrow \infty}\left\|T_{n} v_{n}-W_{n} \widetilde{v}_{n}\right\|=0
$$


On the other hand, for $p \in \Omega$, we find that

$$
\begin{aligned}
\| u_{n} & -p \|^{2} \\
& =\left\|S_{r_{n}}^{(\Theta, \varphi)}\left(I-r_{n} A\right) x_{n}-S_{r_{n}}^{(\Theta, \varphi)}\left(I-r_{n} A\right) p\right\|^{2} \\
& \leq\left\|\left(I-r_{n} A\right) x_{n}-\left(I-r_{n} A\right) p\right\|^{2} \\
& =\left\|x_{n}-p-r_{n}\left(A x_{n}-A p\right)\right\|^{2} \\
& \leq\left\|x_{n}-p\right\|^{2}+r_{n}\left(r_{n}-2 \zeta\right)\left\|A x_{n}-A p\right\|^{2} .
\end{aligned}
$$

Then, together with (61) and (86), we have

$$
\begin{aligned}
\left\|x_{n+1}-p\right\|^{2} & \\
\leq & \left\|v_{n}-p\right\|^{2}-2 \rho_{n}\left(1-\beta_{n}\right)\left\langle\widetilde{A}_{1} v_{n}, \widetilde{v}_{n}-p\right\rangle \\
& \quad-\beta_{n}\left(1-\beta_{n}\right)\left\|T_{n} v_{n}-W_{n} \widetilde{v}_{n}\right\|^{2}-2 \mu \alpha_{n}\left\langle\widetilde{A}_{2} p, x_{n+1}-p\right\rangle \\
\leq & \left\|u_{n}-p\right\|^{2}+2 \rho_{n}\left\|\widetilde{A}_{1} v_{n}\right\|\left\|\widetilde{v}_{n}-p\right\| \\
& +2 \mu \alpha_{n}\left\|\widetilde{A}_{2} p\right\|\left\|x_{n+1}-p\right\| \\
\leq & \left\|x_{n}-p\right\|^{2}+r_{n}\left(r_{n}-2 \zeta\right)\left\|A x_{n}-A p\right\|^{2} \\
& +2 \rho_{n}\left\|\widetilde{A}_{1} v_{n}\right\|\left\|\widetilde{v}_{n}-p\right\|+2 \mu \alpha_{n}\left\|\widetilde{A}_{2} p\right\|\left\|x_{n+1}-p\right\|,
\end{aligned}
$$

which immediately yields

$$
\begin{aligned}
& r_{n}\left(2 \zeta-r_{n}\right)\left\|A x_{n}-A p\right\|^{2} \\
& \leq\left\|x_{n}-p\right\|^{2}-\left\|x_{n+1}-p\right\|^{2}+2 \rho_{n}\left\|\widetilde{A}_{1} v_{n}\right\|\left\|\widetilde{v}_{n}-p\right\| \\
& \quad+2 \mu \alpha_{n}\left\|\widetilde{A}_{2} p\right\|\left\|x_{n+1}-p\right\| \\
& \leq\left\|x_{n}-x_{n+1}\right\|\left(\left\|x_{n}-p\right\|+\left\|x_{n+1}-p\right\|\right) \\
& \quad+2 \rho_{n}\left\|\widetilde{A}_{1} v_{n}\right\|\left\|\widetilde{v}_{n}-p\right\|+2 \mu \alpha_{n}\left\|\widetilde{A}_{2} p\right\|\left\|x_{n+1}-p\right\| .
\end{aligned}
$$

Since $\alpha_{n} \rightarrow 0, \rho_{n} \rightarrow 0,\left\|x_{n+1}-x_{n}\right\| \rightarrow 0$, and $\left\{x_{n}\right\},\left\{v_{n}\right\},\left\{\widetilde{v}_{n}\right\}$ are bounded sequences, it follows from $\left\{r_{n}\right\}_{n=1}^{\infty} \subset[c, d] \subset$ $(0,2 \zeta)$ that

$$
\lim _{n \rightarrow \infty}\left\|A x_{n}-A p\right\|=0
$$

Furthermore, from the firm nonexpansivity of $S_{r_{n}}^{(\Theta, \varphi)}$, we have

$$
\begin{aligned}
& \left\|u_{n}-p\right\|^{2} \\
& \quad=\left\|S_{r_{n}}^{(\Theta, \varphi)}\left(I-r_{n} A\right) x_{n}-S_{r_{n}}^{(\Theta, \varphi)}\left(I-r_{n} A\right) p\right\|^{2}
\end{aligned}
$$

$$
\begin{aligned}
& \leq\left\langle\left(I-r_{n} A\right) x_{n}-\left(I-r_{n} A\right) p, u_{n}-p\right\rangle \\
& =\frac{1}{2}\left[\left\|\left(I-r_{n} A\right) x_{n}-\left(I-r_{n} A\right) p\right\|^{2}+\left\|u_{n}-p\right\|^{2}\right. \\
& \left.\quad \quad-\left\|\left(I-r_{n} A\right) x_{n}-\left(I-r_{n} A\right) p-\left(u_{n}-p\right)\right\|^{2}\right] \\
& \leq \frac{1}{2}\left[\left\|x_{n}-p\right\|^{2}+\left\|u_{n}-p\right\|^{2}\right. \\
& \left.\quad-\left\|x_{n}-u_{n}-r_{n}\left(A x_{n}-A p\right)\right\|^{2}\right] \\
& =\frac{1}{2}\left[\left\|x_{n}-p\right\|^{2}+\left\|u_{n}-p\right\|^{2}-\left\|x_{n}-u_{n}\right\|^{2}\right. \\
& \left.\quad+2 r_{n}\left\langle A x_{n}-A p, x_{n}-u_{n}\right\rangle-r_{n}^{2}\left\|A x_{n}-A p\right\|^{2}\right],
\end{aligned}
$$

which leads to

$$
\begin{aligned}
\left\|u_{n}-p\right\|^{2} \leq & \left\|x_{n}-p\right\|^{2}-\left\|x_{n}-u_{n}\right\|^{2} \\
& +2 r_{n}\left\|A x_{n}-A p\right\|\left\|x_{n}-u_{n}\right\| .
\end{aligned}
$$

From (61), (86), and (94), we have

$$
\begin{aligned}
& \left\|x_{n+1}-p\right\|^{2} \\
& \leq\left\|v_{n}-p\right\|^{2}-2 \rho_{n}\left(1-\beta_{n}\right)\left\langle\widetilde{A}_{1} v_{n}, \widetilde{v}_{n}-p\right\rangle \\
& \quad-\beta_{n}\left(1-\beta_{n}\right)\left\|T_{n} v_{n}-W_{n} \widetilde{v}_{n}\right\|^{2}-2 \mu \alpha_{n}\left\langle\widetilde{A}_{2} p, x_{n+1}-p\right\rangle \\
& \leq\left\|u_{n}-p\right\|^{2}+2 \rho_{n}\left\|\widetilde{A}_{1} v_{n}\right\|\left\|\widetilde{v}_{n}-p\right\| \\
& \quad+2 \mu \alpha_{n}\left\|\widetilde{A}_{2} p\right\|\left\|x_{n+1}-p\right\| \\
& \leq\left\|x_{n}-p\right\|^{2}-\left\|x_{n}-u_{n}\right\|^{2} \\
& \quad+2 r_{n}\left\|A x_{n}-A p\right\|\left\|x_{n}-u_{n}\right\| \\
& \quad+2 \rho_{n}\left\|\widetilde{A}_{1} v_{n}\right\|\left\|\widetilde{v}_{n}-p\right\|+2 \mu \alpha_{n}\left\|\widetilde{A}_{2} p\right\|\left\|x_{n+1}-p\right\|,
\end{aligned}
$$

which hence yields

$$
\begin{aligned}
\| x_{n}- & u_{n} \|^{2} \\
\leq & \left\|x_{n}-p\right\|^{2}-\left\|x_{n+1}-p\right\|^{2} \\
& +2 r_{n}\left\|A x_{n}-A p\right\|\left\|x_{n}-u_{n}\right\| \\
& +2 \rho_{n}\left\|\widetilde{A}_{1} v_{n}\right\|\left\|\widetilde{v}_{n}-p\right\|+2 \mu \alpha_{n}\left\|\widetilde{A}_{2} p\right\|\left\|x_{n+1}-p\right\| \\
\leq & \left\|x_{n}-x_{n+1}\right\|\left(\left\|x_{n}-p\right\|+\left\|x_{n+1}-p\right\|\right) \\
& +2 r_{n}\left\|A x_{n}-A p\right\|\left\|x_{n}-u_{n}\right\| \\
& +2 \rho_{n}\left\|\widetilde{A}_{1} v_{n}\right\|\left\|\widetilde{v}_{n}-p\right\|+2 \mu \alpha_{n}\left\|\widetilde{A}_{2} p\right\|\left\|x_{n+1}-p\right\| .
\end{aligned}
$$

Since $\alpha_{n} \rightarrow 0, \rho_{n} \rightarrow 0,\left\|x_{n+1}-x_{n}\right\| \quad \rightarrow \quad 0$ and $\left\{x_{n}\right\},\left\{u_{n}\right\},\left\{v_{n}\right\},\left\{\widetilde{v}_{n}\right\}$ are bounded sequences, it follows from (92) and $\left\{r_{n}\right\}_{n=1}^{\infty} \subset[c, d] \subset(0,2 \zeta)$ that

$$
\lim _{n \rightarrow \infty}\left\|x_{n}-u_{n}\right\|=0 \text {. }
$$


Next we show that $\lim _{n \rightarrow \infty}\left\|A_{k} \Lambda_{n}^{k} u_{n}-A_{k} p\right\|=0, k=$ $1,2, \ldots, M$. Observe that

$$
\begin{aligned}
& \left\|\Lambda_{n}^{k} u_{n}-p\right\|^{2} \\
& \quad=\left\|J_{R_{k}, \lambda_{k, n}}\left(I-\lambda_{k, n} B_{k}\right) \Lambda_{n}^{k-1} u_{n}-J_{R_{k}, \lambda_{k, n}}\left(I-\lambda_{k, n} B_{k}\right) p\right\|^{2} \\
& \quad \leq\left\|\left(I-\lambda_{k, n} B_{k}\right) \Lambda_{n}^{k-1} u_{n}-\left(I-\lambda_{k, n} B_{k}\right) p\right\|^{2} \\
& \quad \leq\left\|\Lambda_{n}^{k-1} u_{n}-p\right\|^{2}+\lambda_{k, n}\left(\lambda_{k, n}-2 \eta_{i}\right)\left\|B_{k} \Lambda_{n}^{k-1} u_{n}-B_{k} p\right\|^{2} \\
& \quad \leq\left\|u_{n}-p\right\|^{2}+\lambda_{k, n}\left(\lambda_{k, n}-2 \eta_{k}\right)\left\|B_{k} \Lambda_{n}^{k-1} u_{n}-B_{k} p\right\|^{2} \\
& \quad \leq\left\|x_{n}-p\right\|^{2}+\lambda_{k, n}\left(\lambda_{k, n}-2 \eta_{k}\right)\left\|B_{k} \Lambda_{n}^{k-1} u_{n}-B_{k} p\right\|^{2} .
\end{aligned}
$$

Combining (86) and (98), we get

$$
\begin{aligned}
&\left\|x_{n+1}-p\right\|^{2} \\
& \leq\left\|v_{n}-p\right\|^{2}-2 \rho_{n}\left(1-\beta_{n}\right)\left\langle\widetilde{A}_{1} v_{n}, \widetilde{v}_{n}-p\right\rangle \\
& \quad-\beta_{n}\left(1-\beta_{n}\right)\left\|T_{n} v_{n}-W_{n} \widetilde{v}_{n}\right\|^{2}-2 \mu \alpha_{n}\left\langle\widetilde{A}_{2} p, x_{n+1}-p\right\rangle \\
& \leq\left\|\Lambda_{n}^{k} u_{n}-p\right\|^{2}+2 \rho_{n}\left\|\widetilde{A}_{1} v_{n}\right\|\left\|\widetilde{v}_{n}-p\right\| \\
&+2 \mu \alpha_{n}\left\|\widetilde{A}_{2} p\right\|\left\|x_{n+1}-p\right\| \\
& \leq\left\|x_{n}-p\right\|^{2}+\lambda_{k, n}\left(\lambda_{k, n}-2 \eta_{k}\right)\left\|B_{k} \Lambda_{n}^{k-1} u_{n}-B_{k} p\right\|^{2} \\
&+2 \rho_{n}\left\|\widetilde{A}_{1} v_{n}\right\|\left\|\widetilde{v}_{n}-p\right\|+2 \mu \alpha_{n}\left\|\widetilde{A}_{2} p\right\|\left\|x_{n+1}-p\right\|,
\end{aligned}
$$

which hence yields

$$
\begin{aligned}
\lambda_{k, n} & \left(2 \eta_{k}-\lambda_{k, n}\right)\left\|B_{k} \Lambda_{n}^{k-1} u_{n}-B_{k} p\right\|^{2} \\
\leq & \left\|x_{n}-p\right\|^{2}-\left\|x_{n+1}-p\right\|^{2}+2 \rho_{n}\left\|\widetilde{A}_{1} v_{n}\right\|\left\|\widetilde{v}_{n}-p\right\| \\
& +2 \mu \alpha_{n}\left\|\widetilde{A}_{2} p\right\|\left\|x_{n+1}-p\right\| \\
\leq & \left\|x_{n}-x_{n+1}\right\|\left(\left\|x_{n}-p\right\|+\left\|x_{n+1}-p\right\|\right) \\
& +2 \rho_{n}\left\|\widetilde{A}_{1} v_{n}\right\|\left\|\widetilde{v}_{n}-p\right\|+2 \mu \alpha_{n}\left\|\widetilde{A}_{2} p\right\|\left\|x_{n+1}-p\right\| .
\end{aligned}
$$

Since $\alpha_{n} \rightarrow 0, \rho_{n} \rightarrow 0,\left\|x_{n+1}-x_{n}\right\| \rightarrow 0$ and $\left\{x_{n}\right\},\left\{v_{n}\right\},\left\{\widetilde{v}_{n}\right\}$ are bounded sequences, it follows from $\left\{\lambda_{k, n}\right\} \subset\left[a_{k}, b_{k}\right] \subset$ $\left(0,2 \eta_{k}\right), \forall k \in\{1,2, \ldots, M\}$ that

$\lim _{n \rightarrow \infty}\left\|B_{k} \Lambda_{n}^{k-1} u_{n}-B_{k} p\right\|=0, \quad \forall k \in\{1,2, \ldots, M\}$.
By Lemma 11 (a) and Lemma 20, we obtain

$$
\begin{gathered}
\left\|\Lambda_{n}^{k} u_{n}-p\right\|^{2} \\
=\left\|J_{R_{k}, \lambda_{k, n}}\left(I-\lambda_{k, n} B_{k}\right) \Lambda_{n}^{k-1} u_{n}-J_{R_{k}, \lambda_{k, n}}\left(I-\lambda_{k, n} B_{k}\right) p\right\|^{2} \\
\leq\left\langle\left(I-\lambda_{k, n} B_{k}\right) \Lambda_{n}^{k-1} u_{n}-\left(I-\lambda_{k, n} B_{k}\right) p, \Lambda_{n}^{k} u_{n}-p\right\rangle \\
=\frac{1}{2}\left(\left\|\left(I-\lambda_{k, n} B_{k}\right) \Lambda_{n}^{k-1} u_{n}-\left(I-\lambda_{k, n} B_{k}\right) p\right\|^{2}\right. \\
\quad+\left\|\Lambda_{n}^{k} u_{n}-p\right\|^{2} \\
\quad-\|\left(I-\lambda_{k, n} B_{k}\right) \Lambda_{n}^{k-1} u_{n}-\left(I-\lambda_{k, n} B_{k}\right) p \\
\left.\quad-\left(\Lambda_{n}^{k} u_{n}-p\right) \|^{2}\right) \\
\leq \frac{1}{2}\left(\left\|\Lambda_{n}^{k-1} u_{n}-p\right\|^{2}+\left\|\Lambda_{n}^{k} u_{n}-p\right\|^{2}\right. \\
\left.\quad-\left\|\Lambda_{n}^{k-1} u_{n}-\Lambda_{n}^{k} u_{n}-\lambda_{k, n}\left(B_{k} \Lambda_{n}^{k-1} u_{n}-B_{k} p\right)\right\|^{2}\right) \\
\leq \frac{1}{2}\left(\left\|u_{n}-p\right\|^{2}+\left\|\Lambda_{n}^{k} u_{n}-p\right\|^{2}\right. \\
\left.\quad-\left\|\Lambda_{n}^{k-1} u_{n}-\Lambda_{n}^{k} u_{n}-\lambda_{k, n}\left(B_{k} \Lambda_{n}^{k-1} u_{n}-B_{k} p\right)\right\|^{2}\right) \\
\leq \frac{1}{2}\left(\left\|x_{n}-p\right\|^{2}+\left\|\Lambda_{n}^{k} u_{n}-p\right\|^{2}\right. \\
\left.\quad-\left\|\Lambda_{n}^{k-1} u_{n}-\Lambda_{n}^{k} u_{n}-\lambda_{k, n}\left(B_{k} \Lambda_{n}^{k-1} u_{n}-B_{k} p\right)\right\|^{2}\right),
\end{gathered}
$$

which implies

$$
\begin{aligned}
&\left\|\Lambda_{n}^{k} u_{n}-p\right\|^{2} \\
& \leq\left\|x_{n}-p\right\|^{2}-\left\|\Lambda_{n}^{k-1} u_{n}-\Lambda_{n}^{k} u_{n}-\lambda_{k, n}\left(B_{k} \Lambda_{n}^{k-1} u_{n}-B_{k} p\right)\right\|^{2} \\
&=\left\|x_{n}-p\right\|^{2}-\left\|\Lambda_{n}^{k-1} u_{n}-\Lambda_{n}^{k} u_{n}\right\|^{2} \\
& \quad-\lambda_{k, n}^{2}\left\|B_{k} \Lambda_{n}^{k-1} u_{n}-B_{k} p\right\|^{2} \\
&+2 \lambda_{k, n}\left\langle\Lambda_{n}^{k-1} u_{n}-\Lambda_{n}^{k} u_{n}, B_{k} \Lambda_{n}^{k-1} u_{n}-B_{k} p\right\rangle \\
& \leq\left\|x_{n}-p\right\|^{2}-\left\|\Lambda_{n}^{k-1} u_{n}-\Lambda_{n}^{k} u_{n}\right\|^{2} \\
&+2 \lambda_{k, n}\left\|\Lambda_{n}^{k-1} u_{n}-\Lambda_{n}^{k} u_{n}\right\|\left\|B_{k} \Lambda_{n}^{k-1} u_{n}-B_{k} p\right\| .
\end{aligned}
$$

Combining (86) and (103) we conclude that

$$
\begin{aligned}
\| x_{n+1} & -p \|^{2} \\
\leq & \left\|v_{n}-p\right\|^{2}-2 \rho_{n}\left(1-\beta_{n}\right)\left\langle\widetilde{A}_{1} v_{n}, \widetilde{v}_{n}-p\right\rangle \\
& -\beta_{n}\left(1-\beta_{n}\right)\left\|T_{n} v_{n}-W_{n} \widetilde{v}_{n}\right\|^{2} \\
& -2 \mu \alpha_{n}\left\langle\widetilde{A}_{2} p, x_{n+1}-p\right\rangle
\end{aligned}
$$




$$
\begin{aligned}
\leq & \left\|\Lambda_{n}^{k} u_{n}-p\right\|^{2}+2 \rho_{n}\left\|\widetilde{A}_{1} v_{n}\right\|\left\|\widetilde{v}_{n}-p\right\| \\
& +2 \mu \alpha_{n}\left\|\widetilde{A}_{2} p\right\|\left\|x_{n+1}-p\right\| \\
\leq & \left\|x_{n}-p\right\|^{2}-\left\|\Lambda_{n}^{k-1} u_{n}-\Lambda_{n}^{k} u_{n}\right\|^{2} \\
& +2 \lambda_{k, n}\left\|\Lambda_{n}^{k-1} u_{n}-\Lambda_{n}^{k} u_{n}\right\|\left\|B_{k} \Lambda_{n}^{k-1} u_{n}-B_{k} p\right\| \\
& +2 \rho_{n}\left\|\widetilde{A}_{1} v_{n}\right\|\left\|\widetilde{v}_{n}-p\right\|+2 \mu \alpha_{n}\left\|\widetilde{A}_{2} p\right\|\left\|x_{n+1}-p\right\|,
\end{aligned}
$$

which implies

$$
\begin{aligned}
& \left\|\Lambda_{n}^{k-1} u_{n}-\Lambda_{n}^{k} u_{n}\right\|^{2} \\
& \leq\left\|x_{n}-p\right\|^{2}-\left\|x_{n+1}-p\right\|^{2} \\
& \quad+2 \lambda_{k, n}\left\|\Lambda_{n}^{k-1} u_{n}-\Lambda_{n}^{k} u_{n}\right\|\left\|B_{k} \Lambda_{n}^{k-1} u_{n}-B_{k} p\right\| \\
& \quad+2 \rho_{n}\left\|\widetilde{A}_{1} v_{n}\right\|\left\|\widetilde{v}_{n}-p\right\|+2 \mu \alpha_{n}\left\|\widetilde{A}_{2} p\right\|\left\|x_{n+1}-p\right\| \\
& \leq\left\|x_{n}-x_{n+1}\right\|\left(\left\|x_{n}-p\right\|+\left\|x_{n+1}-p\right\|\right) \\
& \quad+2 \lambda_{k, n}\left\|\Lambda_{n}^{k-1} u_{n}-\Lambda_{n}^{k} u_{n}\right\|\left\|B_{k} \Lambda_{n}^{k-1} u_{n}-B_{k} p\right\| \\
& \quad+2 \rho_{n}\left\|\widetilde{A}_{1} v_{n}\right\|\left\|\widetilde{v}_{n}-p\right\|+2 \mu \alpha_{n}\left\|\widetilde{A}_{2} p\right\|\left\|x_{n+1}-p\right\| .
\end{aligned}
$$

Since $\alpha_{n} \rightarrow 0, \rho_{n} \rightarrow 0,\left\|x_{n+1}-x_{n}\right\| \rightarrow 0$, and $\left\{x_{n}\right\},\left\{u_{n}\right\},\left\{v_{n}\right\},\left\{\widetilde{v}_{n}\right\}$ are bounded sequences, it follows from (101) and $\left\{\lambda_{k, n}\right\} \subset\left[a_{k}, b_{k}\right] \subset\left(0,2 \eta_{k}\right), \forall k \in\{1,2, \ldots, M\}$ that

$$
\lim _{n \rightarrow \infty}\left\|\Lambda_{n}^{k-1} u_{n}-\Lambda_{n}^{k} u_{n}\right\|=0, \quad \forall k \in\{1,2, \ldots, M\}
$$

Hence from (106) we get

$$
\begin{aligned}
\left\|u_{n}-v_{n}\right\|= & \left\|\Lambda_{n}^{0} u_{n}-\Lambda_{n}^{N} u_{n}\right\| \\
\leq & \left\|\Lambda_{n}^{0} u_{n}-\Lambda_{n}^{1} u_{n}\right\|+\left\|\Lambda_{n}^{1} u_{n}-\Lambda_{n}^{2} u_{n}\right\| \\
& +\cdots+\left\|\Lambda_{n}^{N-1} u_{n}-\Lambda_{n}^{N} u_{n}\right\| \longrightarrow 0 \\
& \text { as } n \longrightarrow \infty .
\end{aligned}
$$

Thus, from (97) and (107) we obtain

$$
\left\|x_{n}-v_{n}\right\| \leq\left\|x_{n}-u_{n}\right\|+\left\|u_{n}-v_{n}\right\| \longrightarrow 0 \text { as } n \longrightarrow \infty \text {. }
$$
obtain

On the other hand, from (55) and $\left\|x_{n+1}-x_{n}\right\| \rightarrow 0$ we

$$
\begin{aligned}
\left\|x_{n}-y_{n}\right\| & \leq\left\|x_{n}-x_{n+1}\right\|+\left\|x_{n+1}-y_{n}\right\| \\
& \leq\left\|x_{n}-x_{n+1}\right\|+\mu \alpha_{n}\left\|\widetilde{A}_{2} y_{n}\right\| \longrightarrow 0
\end{aligned}
$$

Note that

$$
\begin{aligned}
\left\|T_{n} v_{n}-v_{n}\right\| \leq & \left\|T_{n} v_{n}-y_{n}\right\|+\left\|y_{n}-x_{n}\right\|+\left\|x_{n}-v_{n}\right\| \\
\leq & \left(1-\beta_{n}\right)\left\|W_{n} \widetilde{v}_{n}-T_{n} v_{n}\right\| \\
& \quad+\left\|y_{n}-x_{n}\right\|+\left\|x_{n}-v_{n}\right\| \\
\leq & \left\|W_{n} \widetilde{v}_{n}-T_{n} v_{n}\right\|+\left\|y_{n}-x_{n}\right\|+\left\|x_{n}-v_{n}\right\| .
\end{aligned}
$$

Hence, from (88), (108), and (109) it follows that

$$
\lim _{n \rightarrow \infty}\left\|T_{n} v_{n}-v_{n}\right\|=0
$$

Also, observe that

$$
\begin{aligned}
\left\|P_{C}\left(I-\lambda_{n} \nabla f\right) v_{n}-v_{n}\right\| & =\left\|s_{n} v_{n}+\left(1-s_{n}\right) T_{n} v_{n}-v_{n}\right\| \\
& =\left(1-s_{n}\right)\left\|T_{n} v_{n}-v_{n}\right\| \\
& \leq\left\|T_{n} v_{n}-v_{n}\right\|
\end{aligned}
$$

where $s_{n}=\left(2-\lambda_{n} L\right) / 4 \in(0,1 / 2)$ for each $\lambda_{n} \in(0,2 / L)$. Hence we have

$$
\begin{aligned}
&\left\|P_{C}\left(I-\frac{2}{L} \nabla f\right) v_{n}-v_{n}\right\| \\
& \leq\left\|P_{C}\left(I-\frac{2}{L} \nabla f\right) v_{n}-P_{C}\left(I-\lambda_{n} \nabla f\right) v_{n}\right\| \\
& \quad+\left\|P_{C}\left(I-\lambda_{n} \nabla f\right) v_{n}-v_{n}\right\| \\
& \leq\left\|\left(I-\frac{2}{L} \nabla f\right) v_{n}-\left(I-\lambda_{n} \nabla f\right) v_{n}\right\| \\
& \quad+\left\|P_{C}\left(I-\lambda_{n} \nabla f\right) v_{n}-v_{n}\right\| \\
& \leq\left(\frac{2}{L}-\lambda_{n}\right)\left\|\nabla f\left(v_{n}\right)\right\|+\left\|T_{n} v_{n}-v_{n}\right\| .
\end{aligned}
$$

From the boundedness of $\left\{v_{n}\right\}, s_{n} \rightarrow 0\left(\Leftrightarrow \lambda_{n} \rightarrow 2 / L\right)$ and $\left\|T_{n} v_{n}-v_{n}\right\| \rightarrow 0$ (due to (111)), it follows that

$$
\lim _{n \rightarrow \infty}\left\|v_{n}-P_{C}\left(I-\frac{2}{L} \nabla f\right) v_{n}\right\|=0
$$

Since $\left\|\widetilde{v}_{n}-x_{n}\right\| \leq\left\|v_{n}-x_{n}\right\|+\rho_{n}\left\|\widetilde{A}_{1} v_{n}\right\|$, from (108) and $\rho_{n} \rightarrow 0$, we get

$$
\lim _{n \rightarrow \infty}\left\|\widetilde{v}_{n}-x_{n}\right\|=0
$$

So, from (88), (111), and $\rho_{n} \rightarrow 0$ we deduce that

$$
\begin{aligned}
& \left\|W_{n} \widetilde{v}_{n}-\widetilde{v}_{n}\right\| \\
& \quad \leq\left\|W_{n} \widetilde{v}_{n}-T_{n} v_{n}\right\|+\left\|T_{n} v_{n}-v_{n}\right\|+\left\|v_{n}-\widetilde{v}_{n}\right\| \\
& \quad=\left\|W_{n} \widetilde{v}_{n}-T_{n} v_{n}\right\|+\left\|T_{n} v_{n}-v_{n}\right\|+\rho_{n}\left\|\widetilde{A}_{1} v_{n}\right\| \longrightarrow 0
\end{aligned}
$$


Step 4. We prove that $\omega_{w}\left(x_{n}\right) \subset \Omega$.

Indeed, since $H$ is reflexive and $\left\{x_{n}\right\}$ is bounded, there exists at least a weak convergence subsequence of $\left\{x_{n}\right\}$. Hence it is known that $\omega_{w}\left(x_{n}\right) \neq \emptyset$. Now, take an arbitrary $w \in$ $\omega_{w}\left(x_{n}\right)$. Then there exists a subsequence $\left\{x_{n_{i}}\right\}$ of $\left\{x_{n}\right\}$ such that $x_{n_{i}} \rightarrow w$. From (97), (106), (108), and (115), we have that $u_{n_{i}} \rightarrow$ $w, v_{n_{i}} \rightarrow w, \widetilde{v}_{n_{i}} \rightarrow w$ and $\Lambda_{n_{i}}^{m} u_{n_{i}} \rightarrow w$ for $m=1,2, \ldots, M$. Utilizing Lemma 15, we deduce from $v_{n_{i}} \rightarrow w$ and (114) that $w \in \operatorname{Fix}\left(P_{C}(I-(2 / L) \nabla f)\right)=\operatorname{VI}(C, \nabla f) \stackrel{n_{i}}{=} \Gamma$. Now, let us show that $w \in \cap_{l=1}^{N} \operatorname{Fix}\left(S_{l}\right)$. To see this, we observe that we may assume that (by passing to a further subsequence if necessary)

$$
\mu_{l, n_{i}} \longrightarrow \mu_{l} \in(0,1) \quad(l=1,2, \ldots, N) .
$$

Let $W$ be the $W$-mapping generated by $S_{1}, S_{2}, \ldots, S_{N}$ and $\mu_{1}, \mu_{2}, \ldots, \mu_{N}$. Then, by Proposition 9, we have, for every $x \in$ $H$,

$$
W_{n_{i}} x \longrightarrow W x \quad \text { as } i \longrightarrow \infty \text {. }
$$

Moreover, from Proposition 8 it follows that $\operatorname{Fix}(W)=$ $\cap_{l=1}^{N} \operatorname{Fix}\left(S_{l}\right)$. Assume that $w \notin \cap_{l=1}^{N} \operatorname{Fix}\left(S_{l}\right)$; then, $w \neq W w$. Since $\widetilde{v}_{n_{i}} \rightarrow w$ and $\left\|\widetilde{v}_{n}-W_{n} \widetilde{v}_{n}\right\| \rightarrow 0$ (due to (116)), in terms of Opial's property of a Hilbert space, we conclude from (118) that

$$
\begin{aligned}
& \liminf _{i \rightarrow \infty}\left\|\widetilde{v}_{n_{i}}-w\right\| \\
& <\liminf _{i \rightarrow \infty}\left\|\widetilde{v}_{n_{i}}-W w\right\| \\
& \leq \liminf _{i \rightarrow \infty}\left(\left\|\widetilde{v}_{n_{i}}-W_{n_{i}} \widetilde{v}_{n_{i}}\right\|+\left\|W_{n_{i}} \widetilde{v}_{n_{i}}-W_{n_{i}} w\right\|\right. \\
& \left.\quad+\left\|W_{n_{i}} w-W w\right\|\right) \\
& =\liminf _{i \rightarrow \infty}\left\|W_{n_{i}} \widetilde{v}_{n_{i}}-W_{n_{i}} w\right\| \leq \liminf _{i \rightarrow \infty}\left\|\widetilde{v}_{n_{i}}-w\right\| .
\end{aligned}
$$

This is a contradiction. So, we get $w \in \cap_{l=1}^{N} \operatorname{Fix}\left(S_{l}\right)$. Next, we prove that $w \in \cap_{m=1}^{M} I\left(B_{m}, R_{m}\right)$. As a matter of fact, since $B_{m}$ is $\eta_{m}$-inverse strongly monotone, $B_{m}$ is a monotone and Lipschitz continuous mapping. It follows from Lemma 23 that $R_{m}+B_{m}$ is maximal monotone. Let $(v, g) \in G\left(R_{m}+B_{m}\right)$, that is, $g-B_{m} v \in R_{m} v$. Again, since $\Lambda_{n}^{m} u_{n}=J_{R_{m}, \lambda_{m, n}}(I-$ $\left.\lambda_{m, n} B_{m}\right) \Lambda_{n}^{m-1} u_{n}, n \geq 1, m \in\{1,2, \ldots, M\}$, we have

$$
\Lambda_{n}^{m-1} u_{n}-\lambda_{m, n} B_{m} \Lambda_{n}^{m-1} u_{n} \in\left(I+\lambda_{m, n} R_{m}\right) \Lambda_{n}^{m} u_{n}
$$

that is,

$$
\frac{1}{\lambda_{m, n}}\left(\Lambda_{n}^{m-1} u_{n}-\Lambda_{n}^{m} u_{n}-\lambda_{m, n} B_{m} \Lambda_{n}^{m-1} u_{n}\right) \in R_{m} \Lambda_{n}^{m} u_{n}
$$

In terms of the monotonicity of $R_{m}$, we get

$$
\begin{aligned}
& \left\langle v-\Lambda_{n}^{m} u_{n}, g-B_{m} v-\frac{1}{\lambda_{m, n}}\right. \\
& \left.\quad \times\left(\Lambda_{n}^{m-1} u_{n}-\Lambda_{n}^{m} u_{n}-\lambda_{m, n} B_{m} \Lambda_{n}^{m-1} u_{n}\right)\right\rangle \geq 0,
\end{aligned}
$$

and hence

$$
\begin{aligned}
\langle v- & \left.\Lambda_{n}^{m} u_{n}, g\right\rangle \\
\geq & \left\langle v-\Lambda_{n}^{m} u_{n}, B_{m} v+\frac{1}{\lambda_{m, n}}\right. \\
& \left.\times\left(\Lambda_{n}^{m-1} u_{n}-\Lambda_{n}^{m} u_{n}-\lambda_{m, n} B_{m} \Lambda_{n}^{m-1} u_{n}\right)\right\rangle \\
= & \left\langle v-\Lambda_{n}^{m} u_{n}, B_{m} v-B_{m} \Lambda_{n}^{m} u_{n}+B_{m} \Lambda_{n}^{m} u_{n}-B_{m} \Lambda_{n}^{m-1} u_{n}\right. \\
\geq & \left\langle v-\frac{1}{\lambda_{m, n}}\left(\Lambda_{n}^{m-1} u_{n}-\Lambda_{n}^{m} u_{n}, B_{m} \Lambda_{n}^{m} u_{n}-B_{m} \Lambda_{n}^{m-1} u_{n}\right\rangle\right. \\
& +\left\langle v-\Lambda_{n}^{m} u_{n}, \frac{1}{\lambda_{m, n}}\left(\Lambda_{n}^{m-1} u_{n}-\Lambda_{n}^{m} u_{n}\right)\right\rangle
\end{aligned}
$$

In particular,

$$
\begin{aligned}
& \left\langle v-\Lambda_{n_{i}}^{m} u_{n_{i}}, g\right\rangle \\
& \geq\left\langle v-\Lambda_{n_{i}}^{m} u_{n_{i}}, B_{m} \Lambda_{n_{i}}^{m} u_{n_{i}}-B_{m} \Lambda_{n_{i}}^{m-1} u_{n_{i}}\right\rangle \\
& \quad+\left\langle v-\Lambda_{n_{i}}^{m} u_{n_{i}}, \frac{1}{\lambda_{m, n_{i}}}\left(\Lambda_{n_{i}}^{m-1} u_{n_{i}}-\Lambda_{n_{i}}^{m} u_{n_{i}}\right)\right\rangle .
\end{aligned}
$$

Since $\left\|\Lambda_{n}^{m} u_{n}-\Lambda_{n}^{m-1} u_{n}\right\| \rightarrow 0$ (due to (106)) and $\| B_{m} \Lambda_{n}^{m} u_{n}-$ $B_{m} \Lambda_{n}^{m-1} u_{n} \| \rightarrow 0$ (due to the Lipschitz continuity of $B_{m}$ ), we conclude from $\Lambda_{n_{i}}^{m} u_{n_{i}} \rightarrow w$ and $\left\{\lambda_{m, n}\right\} \subset\left[a_{m}, b_{m}\right] \subset$ $\left(0,2 \eta_{m}\right), m \in\{1,2, \ldots, M\}$ that

$$
\lim _{i \rightarrow \infty}\left\langle v-\Lambda_{n_{i}}^{m} u_{n_{i}}, g\right\rangle=\langle v-w, g\rangle \geq 0
$$

It follows from the maximal monotonicity of $B_{m}+R_{m}$ that $0 \in\left(R_{m}+B_{m}\right) w$; that is, $w \in I\left(B_{m}, R_{m}\right)$. Therefore, $w \in$ $\cap_{m=1}^{M} I\left(B_{m}, R_{m}\right)$.

Next, we show that $w \in \operatorname{GMEP}(\Theta, \varphi, A)$. In fact, from $u_{n}=S_{r_{n}}^{(\Theta, \varphi)}\left(I-r_{n} A\right) x_{n}$, we know that

$$
\begin{aligned}
& \Theta\left(u_{n}, y\right)+\varphi(y)-\varphi\left(u_{n}\right)+\left\langle A x_{n}, y-u_{n}\right\rangle \\
& \quad+\frac{1}{r_{n}}\left\langle K^{\prime}\left(u_{n}\right)-K^{\prime}\left(x_{n}\right), y-u_{n}\right\rangle \geq 0, \quad \forall y \in C .
\end{aligned}
$$

From (H2) it follows that

$$
\begin{aligned}
\varphi(y) & -\varphi\left(u_{n}\right)+\left\langle A x_{n}, y-u_{n}\right\rangle \\
& +\frac{1}{r_{n}}\left\langle K^{\prime}\left(u_{n}\right)-K^{\prime}\left(x_{n}\right), y-u_{n}\right\rangle \geq \Theta\left(y, u_{n}\right),
\end{aligned}
$$


Replacing $n$ by $n_{i}$, we have

$$
\begin{aligned}
& \varphi(y)-\varphi\left(u_{n_{i}}\right)+\left\langle A x_{n_{i}}, y-u_{n_{i}}\right\rangle \\
&+\left\langle\frac{K^{\prime}\left(u_{n_{i}}\right)-K^{\prime}\left(x_{n_{i}}\right)}{r_{n_{i}}}, y-u_{n_{i}}\right\rangle \geq \Theta\left(y, u_{n_{i}}\right), \\
& \forall y \in C .
\end{aligned}
$$

Put $u_{t}=t y+(1-t) w$ for all $t \in(0,1]$ and $y \in C$. Then, from (128), we have

$$
\begin{aligned}
\left\langle u_{t}-\right. & \left.u_{n_{i}}, A u_{t}\right\rangle \\
\geq & \left\langle u_{t}-u_{n_{i}}, A u_{t}\right\rangle-\varphi\left(u_{t}\right)+\varphi\left(u_{n_{i}}\right)-\left\langle u_{t}-u_{n_{i}}, A x_{n_{i}}\right\rangle \\
& -\left\langle\frac{K^{\prime}\left(u_{n_{i}}\right)-K^{\prime}\left(x_{n_{i}}\right)}{r_{n_{i}}}, u_{t}-u_{n_{i}}\right\rangle+\Theta\left(u_{t}, u_{n_{i}}\right) \\
\geq & \left\langle u_{t}-u_{n_{i}}, A u_{t}-A u_{n_{i}}\right\rangle+\left\langle u_{t}-u_{n_{i}}, A u_{n_{i}}-A x_{n_{i}}\right\rangle \\
& -\varphi\left(u_{t}\right)+\varphi\left(u_{n_{i}}\right)-\left\langle\frac{K^{\prime}\left(u_{n_{i}}\right)-K^{\prime}\left(x_{n_{i}}\right)}{r_{n_{i}}}, u_{t}-u_{n_{i}}\right\rangle \\
& +\Theta\left(u_{t}, u_{n_{i}}\right) .
\end{aligned}
$$

Since $\left\|u_{n_{i}}-x_{n_{i}}\right\| \rightarrow 0$ as $i \rightarrow \infty$, we deduce from the Lipschitz continuity of $A$ and $K^{\prime}$ that $\left\|A u_{n_{i}}-A x_{n_{i}}\right\| \rightarrow 0$ and $\left\|K^{\prime}\left(u_{n_{i}}\right)-K^{\prime}\left(x_{n_{i}}\right)\right\| \rightarrow 0$ as $i \rightarrow \infty$. Furthermore, from the monotonicity of $A$, we have $\left\langle u_{t}-u_{n_{i}}, A u_{t}-A u_{n_{i}}\right\rangle \geq 0$. So, from (H4), the weakly lower semicontinuity of $\varphi,\left(K^{\prime}\left(u_{n_{i}}\right)-\right.$ $\left.K^{\prime}\left(x_{n_{i}}\right)\right) / r_{n_{i}} \rightarrow 0$ and $u_{n_{i}} \rightarrow w$, we have

$\left\langle u_{t}-w, A u_{t}\right\rangle \geq-\varphi\left(u_{t}\right)+\varphi(w)+\Theta\left(u_{t}, w\right), \quad$ as $i \longrightarrow \infty$.

From (H1), (H4), and (130) we also have

$$
\begin{aligned}
0= & \Theta\left(u_{t}, u_{t}\right)+\varphi\left(u_{t}\right)-\varphi\left(u_{t}\right) \\
\leq & t \Theta\left(u_{t}, y\right)+(1-t) \Theta\left(u_{t}, w\right)+t \varphi(y) \\
& +(1-t) \varphi(w)-\varphi\left(u_{t}\right) \\
= & t\left[\Theta\left(u_{t}, y\right)+\varphi(y)-\varphi\left(u_{t}\right)\right] \\
& +(1-t)\left[\Theta\left(u_{t}, w\right)+\varphi(w)-\varphi(w)-\varphi\left(u_{t}\right)\right] \\
\leq & t\left[\Theta\left(u_{t}, y\right)+\varphi(y)-\varphi\left(u_{t}\right)\right] \\
& +(1-t)\left\langle u_{t}-w, A u_{t}\right\rangle \\
= & t\left[\Theta\left(u_{t}, y\right)+\varphi(y)-\varphi\left(u_{t}\right)\right]+(1-t) t\left\langle y-w, A u_{t}\right\rangle
\end{aligned}
$$

and hence

$$
0 \leq \Theta\left(u_{t}, y\right)+\varphi(y)-\varphi\left(u_{t}\right)+(1-t)\left\langle y-w, A u_{t}\right\rangle .
$$

Letting $t \rightarrow 0$, we have, for each $y \in C$,

$$
0 \leq \Theta(w, y)+\varphi(y)-\varphi(w)+\langle A w, y-w\rangle
$$

This implies that $w \in \operatorname{GMEP}(\Theta, \varphi, A)$. Therefore, $w \in$ $\cap_{i=1}^{N} \operatorname{Fix}\left(S_{i}\right) \cap \operatorname{GMEP}(\Theta, \varphi, A) \cap \cap_{k=1}^{M} I\left(B_{k}, R_{k}\right) \cap \Gamma:=\Omega$. This shows that $\omega_{w}\left(x_{n}\right) \subset \Omega$.

Step 5. We prove that $\omega_{w}\left(x_{n}\right) \subset \operatorname{VI}\left(\Omega, \widetilde{A}_{1}\right)$ provided $\| x_{n}-$ $y_{n} \|=o\left(\rho_{n}\right)$ additionally.

Indeed, take an arbitrary $w \in \omega_{w}\left(x_{n}\right)$. Then, there exists a subsequence $\left\{x_{n_{i}}\right\}$ of $\left\{x_{n}\right\}$ such that $x_{n_{i}} \rightarrow w$. Since $A_{1}$ is $\alpha$-inverse strongly monotone, from (55), (62), and (66) we conclude that for all $p \in \Omega$

$$
\begin{aligned}
& \left\|y_{n}-p\right\|^{2} \\
& =\left\|\beta_{n}\left(T_{n} v_{n}-p\right)+\left(1-\beta_{n}\right)\left(W_{n} \widetilde{v}_{n}-p\right)\right\|^{2} \\
& \leq \beta_{n}\left\|T_{n} v_{n}-p\right\|^{2}+\left(1-\beta_{n}\right)\left\|W_{n} \widetilde{v}_{n}-p\right\|^{2} \\
& \leq \beta_{n}\left\|v_{n}-p\right\|^{2}+\left(1-\beta_{n}\right)\left\|\widetilde{v}_{n}-p\right\|^{2} \\
& =\beta_{n}\left\|v_{n}-p\right\|^{2}+\left(1-\beta_{n}\right)\left\|v_{n}-p-\rho_{n} \widetilde{A}_{1} v_{n}\right\|^{2} \\
& \leq \beta_{n}\left\|v_{n}-p\right\|^{2}+\left(1-\beta_{n}\right)\left[\left\|v_{n}-p\right\|^{2}-2 \rho_{n}\left\langle\widetilde{A}_{1} v_{n}, \widetilde{v}_{n}-p\right\rangle\right] \\
& =\beta_{n}\left\|v_{n}-p\right\|^{2}+\left(1-\beta_{n}\right) \\
& \times\left[\left\|v_{n}-p\right\|^{2}-2 \rho_{n}\left(\left\langle\widetilde{A}_{1} v_{n}, v_{n}-p\right\rangle\right.\right. \\
& \left.\left.+\left\langle\widetilde{A}_{1} v_{n}, \widetilde{v}_{n}-v_{n}\right\rangle\right)\right] \\
& =\beta_{n}\left\|v_{n}-p\right\|^{2}+\left(1-\beta_{n}\right) \\
& \times\left[\left\|v_{n}-p\right\|^{2}-2 \rho_{n}\right. \\
& \times\left(\left\langle\widetilde{A}_{1} v_{n}-\widetilde{A}_{1} p, v_{n}-p\right\rangle\right. \\
& \left.\left.+\left\langle\widetilde{A}_{1} p, v_{n}-p\right\rangle+\left\langle\widetilde{A}_{1} v_{n}, \widetilde{v}_{n}-v_{n}\right\rangle\right)\right] \\
& =\left\|v_{n}-p\right\|^{2}-2 \rho_{n}\left(1-\beta_{n}\right) \\
& \times\left(\left\langle\widetilde{A}_{1} v_{n}-\widetilde{A}_{1} p, v_{n}-p\right\rangle\right. \\
& \left.+\left\langle\widetilde{A}_{1} p, v_{n}-p\right\rangle+\left\langle\widetilde{A}_{1} v_{n}, \widetilde{v}_{n}-v_{n}\right\rangle\right) \\
& \leq\left\|x_{n}-p\right\|^{2}-2 \rho_{n}\left(1-\beta_{n}\right)\left\langle\widetilde{A}_{1} p, v_{n}-p\right\rangle \\
& +2 \rho_{n}\left(1-\beta_{n}\right)\left\|\widetilde{A}_{1} v_{n}\right\|\left\|\widetilde{v}_{n}-v_{n}\right\|,
\end{aligned}
$$

which implies that

$$
\left\langle\widetilde{A}_{1} p, v_{n}-p\right\rangle
$$

$$
\leq \frac{1}{2 \rho_{n}\left(1-\beta_{n}\right)}\left(\left\|x_{n}-p\right\|^{2}-\left\|y_{n}-p\right\|^{2}\right)
$$




$$
\begin{aligned}
& +\left\|\widetilde{A}_{1} v_{n}\right\|\left\|\widetilde{v}_{n}-v_{n}\right\| \\
\leq & \frac{\left\|x_{n}-y_{n}\right\|}{2 \rho_{n}\left(1-\beta_{n}\right)}\left(\left\|x_{n}-p\right\|+\left\|y_{n}-p\right\|\right)+\rho_{n}^{2}\left\|\widetilde{A}_{1} v_{n}\right\|^{2} .
\end{aligned}
$$

So, from $\rho_{n} \rightarrow 0$ and the assumption $\left\|x_{n}-y_{n}\right\|=o\left(\rho_{n}\right)$, we get

$$
\limsup _{n \rightarrow \infty}\left\langle\widetilde{A}_{1} p, v_{n}-p\right\rangle \leq 0 .
$$

Thus, it follows from (108) that for all $p \in \Omega$

$$
\begin{aligned}
\left\langle\widetilde{A}_{1} p, w-p\right\rangle & =\lim _{i \rightarrow \infty}\left\langle\widetilde{A}_{1} p, x_{n_{i}}-p\right\rangle \\
& \leq \limsup _{n \rightarrow \infty}\left\langle\widetilde{A}_{1} p, x_{n}-p\right\rangle \\
& =\limsup _{n \rightarrow \infty}\left(\left\langle\widetilde{A}_{1} p, v_{n}-p\right\rangle+\left\langle\widetilde{A}_{1} p, x_{n}-v_{n}\right\rangle\right) \\
& =\limsup _{n \rightarrow \infty}\left\langle\widetilde{A}_{1} p, v_{n}-p\right\rangle \leq 0,
\end{aligned}
$$

that is,

$$
\left\langle\widetilde{A}_{1} p, p-w\right\rangle \geq 0, \quad \forall p \in \Omega .
$$

Since $\widetilde{A}_{1}$ is $\alpha$-inverse strongly monotone, by Minty's Lemma [23] we know that (138) is equivalent to the VIP

$$
\left\langle\widetilde{A}_{1} w, p-w\right\rangle \geq 0, \quad \forall p \in \Omega .
$$

This shows that $w \in \operatorname{VI}\left(\Omega, \widetilde{A}_{1}\right)$. Therefore, $\omega_{w}\left(x_{n}\right) \subset$ $\mathrm{VI}\left(\Omega, \widetilde{A_{1}}\right)$.

Theorem 25. Assume that all the conditions in Theorem 24 are satisfied. Then, we have that

(i) $\left\{x_{n}\right\}$ converges strongly to a point $u^{*} \in \Omega$, which is a unique solution of the VIP

$$
\left\langle\widetilde{A}_{2} u^{*}, p-u^{*}\right\rangle \geq 0, \quad \forall p \in \Omega
$$

(ii) $\left\{x_{n}\right\}$ converges strongly to a unique solution of THVI (16) provided $\left\|x_{n}-y_{n}\right\|=o\left(\rho_{n}\right)$ additionally.

Proof. Since $\widetilde{A}_{2}$ is $\beta$-strongly monotone and L-Lipschitz continuous, there exists a unique solution $u^{*} \in \Omega$ of the VIP

$$
\left\langle\widetilde{A_{2}} u^{*}, p-u^{*}\right\rangle \geq 0, \quad \forall p \in \Omega \text {. }
$$

Now, let us show that

$$
\limsup _{n \rightarrow \infty}\left\langle\widetilde{A}_{2} u^{*}, u^{*}-x_{n}\right\rangle \leq 0 .
$$

Since $\left\{x_{n}\right\}$ is bounded, we may assume, without loss of generality, that there exists a subsequence $\left\{x_{n_{i}}\right\}$ of $\left\{x_{n}\right\}$ such that $x_{n_{i}} \rightarrow w$ and

$$
\begin{aligned}
\limsup _{n \rightarrow \infty}\left\langle\widetilde{A}_{2} u^{*}, u^{*}-x_{n}\right\rangle & =\lim _{i \rightarrow \infty}\left\langle\widetilde{A}_{2} u^{*}, u^{*}-x_{n_{i}}\right\rangle \\
& =\left\langle\widetilde{A}_{2} u^{*}, u^{*}-w\right\rangle .
\end{aligned}
$$

In terms of Theorem 24 (ii), we know that $w \in \omega_{w}\left(x_{n}\right) \subset \Omega$. So, from (141) it follows that

$$
\limsup _{n \rightarrow \infty}\left\langle\widetilde{A}_{2} u^{*}, u^{*}-x_{n}\right\rangle=\left\langle\widetilde{A}_{2} u^{*}, u^{*}-w\right\rangle \leq 0 .
$$

Next, let us show that $\lim _{n \rightarrow \infty}\left\|x_{n}-u^{*}\right\|=0$. In fact, by utilizing Lemma 10, from (55) and (134) with $p=u^{*}$, we get

$$
\begin{aligned}
\left\|x_{n+1}-u^{*}\right\|^{2} & \left\|\left(I-\mu \alpha_{n} \widetilde{A}_{2}\right) y_{n}-u^{*}\right\|^{2} \\
= & \|\left(I-\mu \alpha_{n} \widetilde{A}_{2}\right) y_{n}-\left(I-\mu \alpha_{n} \widetilde{A}_{2}\right) u^{*} \\
& +\left(I-\mu \alpha_{n} \widetilde{A}_{2}\right) u^{*}-u^{*} \|^{2} \\
\leq & \left\|\left(I-\mu \alpha_{n} \widetilde{A}_{2}\right) y_{n}-\left(I-\mu \alpha_{n} \widetilde{A}_{2}\right) u^{*}\right\|^{2} \\
& -2 \mu \alpha_{n}\left\langle\widetilde{A}_{2} u^{*}, x_{n+1}-u^{*}\right\rangle \\
\leq & \left(1-\alpha_{n} \tau\right)\left\|y_{n}-u^{*}\right\|^{2}-2 \mu \alpha_{n}\left\langle\widetilde{A}_{2} u^{*}, x_{n+1}-u^{*}\right\rangle \\
\leq & \left(1-\alpha_{n} \tau\right)\left[\left\|x_{n}-u^{*}\right\|^{2}-2 \rho_{n}\left(1-\beta_{n}\right)\left\langle\widetilde{A}_{1} u^{*}, v_{n}-u^{*}\right\rangle\right. \\
& \left.\quad+2 \rho_{n}\left(1-\beta_{n}\right)\left\|\widetilde{A}_{1} v_{n}\right\|\left\|\widetilde{v}_{n}-v_{n}\right\|\right] \\
= & \quad\left(1-\alpha_{n} \tau\right)\left\|x_{n}-u^{*}\right\|^{2} \cdot \frac{2}{\tau}\left\{\frac{\rho_{n}}{\alpha_{n}}\left\|\widetilde{A}_{1} u^{*}\right\|\left\|v_{n}-u^{*}\right\|+\frac{\rho_{n}^{2}}{\alpha_{n}}\left\|\widetilde{A}_{1} v_{n}\right\|^{2}\right. \\
& -2 \mu \alpha_{n}\left\langle\widetilde{A}_{2} u^{*}, x_{n+1}-u^{*}\right\rangle \\
& \left.+2 \alpha_{n} \tau\right)\left\|x_{n}-u^{*}\right\|^{2} \\
& +2 \mu \alpha_{n}\left\langle\widetilde{A}_{2} u^{*}\|\| v_{n}-u^{*}\left\|+2 \rho_{n}^{2}\right\| \widetilde{A}_{1} v_{n} \|^{2}\right.
\end{aligned}
$$

where $\tau=1-\sqrt{1-\mu\left(2 \beta-\mu \kappa^{2}\right)}$.

Since $\sum_{n=1}^{\infty} \alpha_{n}=\infty, \lim _{n \rightarrow \infty}\left(\rho_{n} / \alpha_{n}\right)=0$, and $\lim \sup _{n \rightarrow \infty}\left\langle\widetilde{A}_{2} u^{*}, u^{*}-x_{n+1}\right\rangle \leq 0$ (due to (144)), we deduce that $\sum_{n=1}^{\infty} \alpha_{n} \tau=\infty$ and

$$
\begin{aligned}
\limsup _{n \rightarrow \infty} \frac{2}{\tau}\left\{\frac{\rho_{n}}{\alpha_{n}}\left\|\widetilde{A}_{1} u^{*}\right\|\right. & \left\|v_{n}-u^{*}\right\|+\frac{\rho_{n}^{2}}{\alpha_{n}}\left\|\widetilde{A}_{1} v_{n}\right\|^{2} \\
& \left.+\mu\left\langle\widetilde{A}_{2} u^{*}, u^{*}-x_{n+1}\right\rangle\right\} \leq 0 .
\end{aligned}
$$

Therefore, by applying Lemma 18 to (145) we infer that $\lim _{n \rightarrow \infty}\left\|x_{n}-u^{*}\right\|=0$. 
Finally, we prove that $\lim _{n \rightarrow \infty}\left\|x_{n}-x^{*}\right\|=0$ provided $\left\|x_{n}-y_{n}\right\|=o\left(\alpha_{n}\right)$ additionally, where $\left\{x^{*}\right\}=$ $\operatorname{VI}\left(\operatorname{VI}\left(\Omega, \widetilde{A}_{1}\right), \widetilde{A}_{2}\right)$.

Indeed, first of all, let us show that $\omega_{w}\left(x_{n}\right)=\left\{x^{*}\right\}$. As a matter of fact, take an arbitrary $w \in \omega_{w}\left(x_{n}\right)$. Then, there exists a subsequence $\left\{x_{n_{j}}\right\}$ of $\left\{x_{n}\right\}$ such that $x_{n_{j}} \rightarrow w$. Moreover, by Theorem 24 (iii) we know that $w \in \omega_{w}\left(x_{n}\right) \subset \operatorname{VI}\left(\Omega, \widetilde{A}_{1}\right)$. Utilizing Lemmas 10 and 17, from (55) and (134), we deduce that for all $p \in \operatorname{VI}\left(\Omega, \widetilde{A_{1}}\right)$

$$
\begin{aligned}
\left\|x_{n+1}-p\right\|^{2} & \\
= & \left\|\left(I-\mu \alpha_{n} \widetilde{A}_{2}\right) y_{n}-p\right\|^{2} \\
= & \left\|\left(I-\mu \alpha_{n} \widetilde{A}_{2}\right) y_{n}-\left(I-\mu \alpha_{n} \widetilde{A}_{2}\right) p+\left(I-\mu \alpha_{n} \widetilde{A}_{2}\right) p-p\right\|^{2} \\
\leq & \left\|\left(I-\mu \alpha_{n} \widetilde{A}_{2}\right) y_{n}-\left(I-\mu \alpha_{n} \widetilde{A}_{2}\right) p\right\|^{2} \\
& -2 \mu \alpha_{n}\left\langle\widetilde{A}_{2} p, x_{n+1}-p\right\rangle \\
\leq & \left(1-\alpha_{n} \tau\right)\left\|y_{n}-p\right\|^{2}-2 \mu \alpha_{n}\left\langle\widetilde{A}_{2} p, x_{n+1}-p\right\rangle \\
\leq & \left(1-\alpha_{n} \tau\right)\left[\left\|x_{n}-p\right\|^{2}-2 \rho_{n}\left(1-\beta_{n}\right)\left\langle\widetilde{A}_{1} p, v_{n}-p\right\rangle\right. \\
& \quad+2 \mu \alpha_{n}\left\langle\widetilde{A}_{2} p, x_{n+1}-p\right\rangle \\
\leq & \left\|x_{n}-p\right\|^{2}+2 \rho_{n}\left\|\widetilde{A}_{1} p\right\|\left\|v_{n}-p\right\|+2 \rho_{n}^{2}\left\|\widetilde{A}_{1} v_{n}\right\| \|^{2} \\
& -2 \mu \alpha_{n}\left\langle\widetilde{A}_{2}-v_{n} \|\right]
\end{aligned}
$$

where $\tau=1-\sqrt{1-\mu\left(2 \beta-\mu \kappa^{2}\right)}$. So, it follows that

$$
\begin{aligned}
\left\langle\widetilde{A}_{2} p, x_{n+1}-p\right\rangle & \\
\leq & \frac{1}{2 \mu \alpha_{n}}\left(\left\|x_{n}-p\right\|^{2}-\left\|x_{n+1}-p\right\|^{2}\right) \\
& +\frac{\rho_{n}}{\mu \alpha_{n}}\left[\left\|\widetilde{A}_{1} p\right\|\left\|v_{n}-p\right\|+\rho_{n}\left\|\widetilde{A}_{1} v_{n}\right\|^{2}\right] \\
\leq & \frac{\left\|x_{n}-x_{n+1}\right\|}{2 \mu \alpha_{n}}\left(\left\|x_{n}-p\right\|+\left\|x_{n+1}-p\right\|\right) \\
& +\frac{\rho_{n}}{\mu \alpha_{n}}\left[\left\|\widetilde{A}_{1} p\right\|\left\|v_{n}-p\right\|+\rho_{n}\left\|\widetilde{A}_{1} v_{n}\right\|^{2}\right] .
\end{aligned}
$$

Since $\alpha_{n} \rightarrow 0, \rho_{n}=o\left(\alpha_{n}\right)$ and $\left\|x_{n+1}-x_{n}\right\|=o\left(\rho_{n}\right)$, we find that

$$
\lim _{n \rightarrow \infty} \frac{\left\|x_{n+1}-x_{n}\right\|}{\alpha_{n}}=\lim _{n \rightarrow \infty} \frac{\left\|x_{n+1}-x_{n}\right\|}{\rho_{n}} \cdot \frac{\rho_{n}}{\alpha_{n}}=0 .
$$

Hence, we conclude from (148) that for all $p \in \operatorname{VI}\left(\Omega, \widetilde{A_{1}}\right)$

$$
\begin{aligned}
& \left\langle\widetilde{A}_{2} p, w-p\right\rangle \\
& =\lim _{j \rightarrow \infty}\left\langle\widetilde{A}_{2} p, x_{n_{j}}-p\right\rangle \leq \limsup _{n \rightarrow \infty}\left\langle\widetilde{A}_{2} p, x_{n}-p\right\rangle \\
& =\limsup _{n \rightarrow \infty}\left(\left\langle\widetilde{A}_{2} p, x_{n}-x_{n+1}\right\rangle+\left\langle\widetilde{A}_{2} p, x_{n+1}-p\right\rangle\right) \\
& =\limsup _{n \rightarrow \infty}\left\langle\widetilde{A}_{2} p, x_{n+1}-p\right\rangle \leq 0,
\end{aligned}
$$

that is,

$$
\left\langle\widetilde{A}_{2} p, p-w\right\rangle \geq 0, \quad \forall p \in \mathrm{VI}\left(\Omega, \widetilde{A}_{1}\right) .
$$

Since $\widetilde{A}_{2}$ is $\beta$-strongly monotone and $L$-Lipschitz continuous, by Minty's Lemma [23] we know that (151) is equivalent to the VIP

$$
\left\langle\widetilde{A}_{2} w, p-w\right\rangle \geq 0, \quad \forall p \in \mathrm{VI}\left(\Omega, \widetilde{A}_{1}\right) .
$$

This shows that $w \in \operatorname{VI}\left(\operatorname{VI}\left(\Omega, \widetilde{A}_{1}\right), \widetilde{A}_{2}\right)$. Considering $\left\{x^{*}\right\}=$ $\operatorname{VI}\left(\operatorname{VI}\left(\Omega, \widetilde{A}_{1}\right), \widetilde{A}_{2}\right)$, we know that $w=x^{*}$. Thus, $\omega_{w}\left(x_{n}\right)=$ $\left\{x^{*}\right\}$; that is, $x_{n} \rightarrow x^{*}$.

Next we prove that $\lim _{n \rightarrow \infty}\left\|x_{n}-x^{*}\right\|=0$. As a matter of fact, by utilizing (147) with $p=x^{*}$, we get

$$
\begin{aligned}
\left\|x_{n+1}-x^{*}\right\|^{2} & \\
\leq & \left(1-\alpha_{n} \tau\right) \\
& \times\left[\left\|x_{n}-x^{*}\right\|^{2}-2 \rho_{n}\left(1-\beta_{n}\right)\left\langle\widetilde{A}_{1} x^{*}, v_{n}-x^{*}\right\rangle\right. \\
& \left.+2 \rho_{n}\left(1-\beta_{n}\right)\left\|\widetilde{A}_{1} v_{n}\right\|\left\|\widetilde{v}_{n}-v_{n}\right\|\right] \\
& -2 \mu \alpha_{n}\left\langle\widetilde{A}_{2} x^{*}, x_{n+1}-x^{*}\right\rangle \\
\leq & \left(1-\alpha_{n} \tau\right)\left\|x_{n}-x^{*}\right\|^{2} \\
& +2 \rho_{n}\left\|\widetilde{A}_{1} x^{*}\right\|\left\|v_{n}-x^{*}\right\|+2 \rho_{n}^{2}\left\|\widetilde{A}_{1} v_{n}\right\|^{2} \\
& -2 \mu \alpha_{n}\left\langle\widetilde{A}_{2} x^{*}, x_{n+1}-x^{*}\right\rangle \\
= & \left(1-\alpha_{n} \tau\right)\left\|x_{n}-x^{*}\right\|^{2} \\
& +\alpha_{n} \tau \cdot \frac{2}{\tau}\left[\frac{\rho_{n}}{\alpha_{n}}\left\|\widetilde{A}_{1} x^{*}\right\|\left\|v_{n}-x^{*}\right\|\right. \\
& \left.\quad \frac{\rho_{n}^{2}}{\alpha_{n}}\left\|\widetilde{A}_{1} v_{n}\right\|^{2}-\mu\left\langle\widetilde{A}_{2} x^{*}, x_{n+1}-x^{*}\right\rangle\right] .
\end{aligned}
$$

Since $\sum_{n=1}^{\infty} \alpha_{n}=\infty, \lim _{n \rightarrow \infty}\left(\rho_{n} / \alpha_{n}\right)=0$, and $\lim _{n \rightarrow \infty}$ $\left\langle\widetilde{A}_{2} x^{*}, x^{*}-x_{n+1}\right\rangle=0$ (due to $x_{n} \rightarrow x^{*}$ ), we deduce that $\sum_{n=1}^{\infty} \alpha_{n} \tau=\infty$, and

$$
\begin{aligned}
\lim _{n \rightarrow \infty} \frac{2}{\mathcal{\tau}}\left[\frac{\rho_{n}}{\alpha_{n}}\left\|\widetilde{A}_{1} x^{*}\right\|\left\|v_{n}-x^{*}\right\|\right. \\
\left.+\frac{\rho_{n}^{2}}{\alpha_{n}}\left\|\widetilde{A}_{1} v_{n}\right\|^{2}-\mu\left\langle\widetilde{A}_{2} x^{*}, x_{n+1}-x^{*}\right\rangle\right]=0 .
\end{aligned}
$$


Therefore, applying Lemma 18 to (153) we infer that $\lim _{n \rightarrow \infty}\left\|x_{n}-x^{*}\right\|=0$. This completes the proof.

Remark 26. In 2012, Ceng et al. [19] proposed and analyzed the following hybrid iterative method for finding a common element of the set of solutions of GMEP (3) and the set of fixed points of a finite family of nonexpansive mappings $\left\{S_{i}\right\}_{i=1}^{N}$.

Theorem CGY (see [19, Theorem 3.1]). Let $C$ be a nonempty closed convex subset of a real Hilbert space $H$. Let $\Theta: C \times$ $\mathrm{C} \rightarrow \mathbf{R}$ be a bifunction satisfying assumptions (H1)-(H4) and let $\varphi: C \rightarrow \mathbf{R}$ be a lower semicontinuous and convex function with restriction (A1) or (A2). Let the mapping $A$ : $H \rightarrow H$ be $\zeta$-inverse strongly monotone, and let $\left\{S_{i}\right\}_{i=1}^{N}$ be a finite family of nonexpansive mappings on $H$ such that $\left(\cap_{i=1}^{N} \operatorname{Fix}\left(S_{i}\right)\right) \cap \operatorname{GMEP}(\Theta, \varphi, A) \neq \emptyset$. Let $F: H \rightarrow H$ be a $\kappa-$ Lipschitzian and $\eta$-strongly monotone operator with constants $\kappa, \eta>0$ and $V: H \rightarrow H$ a $\rho$-Lipschitzian mapping with a constant $\rho \geq 0$. Let $0<\mu<2 \eta / \kappa^{2}$ and $0 \leq \gamma \rho<\tau$, where $\tau=1-\sqrt{1-\mu\left(2 \eta-\mu \kappa^{2}\right)}$. Suppose $\left\{\alpha_{n}\right\}$ and $\left\{\beta_{n}\right\}$ are two sequences in $(0,1),\left\{r_{n}\right\}$ is a sequence in $(0,2 \zeta]$, and $\left\{\mu_{i, n}\right\}_{i=1}^{N}$ is a sequence in $[a, b]$ with $0<a \leq b<1$. For every $n \geq 1$, let $W_{n}$ be the $W$-mapping generated by $S_{1}, S_{2}, \ldots, S_{N}$ and $\mu_{1, n}, \mu_{2, n}, \ldots, \mu_{N, n}$. Given $x_{1} \in H$ arbitrarily, suppose the sequences $\left\{x_{n}\right\}$ and $\left\{u_{n}\right\}$ are generated iteratively by

$$
\begin{gathered}
\Theta\left(u_{n}, y\right)+\varphi(y)-\varphi\left(u_{n}\right)+\left\langle A x_{n}, y-u_{n}\right\rangle \\
+\frac{1}{r_{n}}\left\langle y-u_{n}, u_{n}-x_{n}\right\rangle \geq 0, \quad \forall y \in C, \\
x_{n+1}=\alpha_{n} \gamma V x_{n}+\beta_{n} x_{n}+\left(\left(1-\beta_{n}\right) I-\alpha_{n} \mu F\right) W_{n} u_{n}, \\
\forall n \geq 1,
\end{gathered}
$$

where the sequences $\left\{\alpha_{n}\right\},\left\{\beta_{n}\right\},\left\{r_{n}\right\}$, and the finite family of sequences $\left\{\mu_{i, n}\right\}_{i=1}^{N}$ satisfy the following conditions:

(i) $\lim _{n \rightarrow \infty} \alpha_{n}=0$ and $\sum_{n=1}^{\infty} \alpha_{n}=\infty$;

(ii) $0<\liminf _{n \rightarrow \infty} \beta_{n} \leq \lim \sup _{n \rightarrow \infty} \beta_{n}<1$;

(iii) $0<\liminf _{n \rightarrow \infty} r_{n} \leq \limsup _{n \rightarrow \infty} r_{n}<2 \zeta$ and $\lim _{n \rightarrow \infty}\left(r_{n+1}-r_{n}\right)=0$;

(iv) $\lim _{n \rightarrow \infty}\left(\mu_{i, n+1}-\mu_{i, n}\right)=0$ for all $i=1,2, \ldots, N$.

Then, both $\left\{x_{n}\right\}$ and $\left\{u_{n}\right\}$ converge strongly to $x^{*} \in \cap_{i=1}^{N} \operatorname{Fix}\left(S_{i}\right) \cap \operatorname{GMEP}(\Theta, \varphi, A)$, where $x^{*}=$ $P_{\cap_{i=1}^{N} \operatorname{Fix}\left(S_{i}\right) \cap G M E P(\Theta, \varphi, A)}(I-\mu F+\gamma V) x^{*}$ is a unique solution of the VIP

$$
\begin{aligned}
& \left\langle(\mu F-\gamma V) x^{*}, p-x^{*}\right\rangle \leq 0, \\
& \forall p \in\left(\bigcap_{i=1}^{N} \operatorname{Fix}\left(S_{i}\right)\right) \cap \operatorname{GMEP}(\Theta, \varphi, A) .
\end{aligned}
$$

It is obvious that our iterative scheme (55) is very different from Yao, Liou, and Marino's iterative one (13), Zeng, Wong, and Yao's iterative one in Algorithm ZWY. and Ceng, Guu. and Yao's iterative one (155). Here, the two-step iterative scheme in [7, Algorithm 3.2] is extended to develop our four-step iterative scheme (55) for the THVI (16) by combining Korpelevich's extragradient method, hybrid steepest-descent method, and averaged mapping approach to the gradient-projection algorithm. The problem of finding a point $x^{*} \in \cap_{i=1}^{N} \operatorname{Fix}\left(S_{i}\right) \cap$ $\operatorname{GMEP}(\Theta, \varphi, A)$ in [19] is extended to the more general problem of finding a point $x^{*} \in\left(\cap_{i=1}^{N} \operatorname{Fix}\left(S_{i}\right)\right) \cap \operatorname{GMEP}(\Theta, \varphi, A) \cap$ $\left(\cap_{k=1}^{M} I\left(B_{k}, R_{k}\right)\right) \cap \Gamma$, which is involved in THVI (16). It is worth pointing out that under the lack of the assumptions similar to those in [6, Theorem 3.2], for example, $\left\{x_{n}\right\}$ is bounded, $\operatorname{Fix}(T) \cap \operatorname{int} C \neq \emptyset$ and $\|x-T x\| \geq k \operatorname{Dist}(x, \operatorname{Fix}(T)), \forall x \in C$ for some $k>0$, the sequence $\left\{x_{n}\right\}$ generated by (55) converges strongly to a point $u^{*} \in\left(\cap_{i=1}^{N} \operatorname{Fix}\left(S_{i}\right)\right) \cap \operatorname{GMEP}(\Theta, \varphi, A) \cap$ $\left(\cap_{k=1}^{M} I\left(B_{k}, R_{k}\right)\right) \cap \Gamma=: \Omega$, which is a unique solution of the $\operatorname{VIP}\left\langle\widetilde{A}_{2} u^{*}, p-u^{*}\right\rangle \geq 0, \forall p \in \Omega$.

\section{Conflict of Interests}

The authors declare that there is no conflict of interests regarding the publication of this paper.

\section{Acknowledgments}

This research was partially supported by the National Science Foundation of China (11071169), Innovation Program of Shanghai Municipal Education Commission (09ZZ133), and Ph.D. Program Foundation of Ministry of Education of China (20123127110002). This work was supported partly by the National Science Council of the Republic of China. This research was partially supported by a grant from NSC.

\section{References}

[1] J. L. Lions, Quelques Méthodes de Résolution des Problèmes aux Limites Non Linéaires, Dunod, Paris, Farnce, 1969.

[2] J. W. Peng and J. C. Yao, "A new hybrid-extragradient method for generalized mixed equilibrium problems, fixed point problems and variational inequality problems," Taiwanese Journal of Mathematics, vol. 12, no. 6, pp. 1401-1432, 2008.

[3] H. K. Xu and T. H. Kim, "Convergence of hybrid steepestdescent methods for variational inequalities," Journal of Optimization Theory and Applications, vol. 119, no. 1, pp. 185-201, 2003.

[4] H. K. Xu, "Averaged mappings and the gradient-projection algorithm," Journal of Optimization Theory and Applications, vol. 150, no. 2, pp. 360-378, 2011.

[5] L. C. Ceng, Q. H. Ansari, and J. C. Yao, "Some iterative methods for finding fixed points and for solving constrained convex minimization problems," Nonlinear Analysis, vol. 74, no. 16, pp. 5286-5302, 2011.

[6] Y. Yao, Y. C. Liou, and G. Marino, “Two-step iterative algorithms for hierarchical fixed point problems and variational inequality problems," Journal of Applied Mathematics and Computing, vol. 31, no. 1-2, pp. 433-445, 2009.

[7] L. C. Zeng, M. M. Wong, and J. C. Yao, "Strong convergence of relaxed hybrid steepest-descent methods for triple hierarchical constrained optimization," Fixed Point Theory and Applications, vol. 2012, articel 29, 24 pages, 2012.

[8] L. Ceng, Q. H. Ansari, and J. Yao, "Relaxed extragradient methods for finding minimum-norm solutions of the split 
feasibility problem," Nonlinear Analysis: Theory, Methods \& Applications, vol. 75, no. 4, pp. 2116-2125, 2012.

[9] L. Ceng, Q. H. Ansari, and J. Yao, "An extragradient method for solving split feasibility and fixed point problems," Computers \& Mathematics with Applications, vol. 64, no. 4, pp. 633-642, 2012.

[10] L.-C. Ceng, S.-M. Guu, and J.-C. Yao, "Hybrid iterative method for finding common solutions of generalized mixed equilibrium and fixed point problems," Fixed Point Theory and Applications, vol. 2012, article 92, 2012.

[11] L. Ceng, Q. H. Ansari, N. Wong, and J. Yao, "An extragradientlike approximation method for variational inequalities and fixed point problems," Fixed Point Theory and Applications, vol. 2011, article 22, 18 pages, 2011.

[12] L. Ceng, Q. H. Ansari, and J. Yao, "Relaxed extragradient iterative methods for variational inequalities," Applied Mathematics and Computation, vol. 218, no. 3, pp. 1112-1123, 2011.

[13] L. C. Ceng, M. Teboulle, and J. C. Yao, "Weak convergence of an iterative method for pseudomonotone variational inequalities and fixed-point problems," Journal of Optimization Theory and Applications, vol. 146, no. 1, pp. 19-31, 2010.

[14] L. C. Ceng, A. Petruşel, and J. C. Yao, "Iterative approaches to solving equilibrium problems and fixed point problems of infinitely many nonexpansive mappings," Journal of Optimization Theory and Applications, vol. 143, no. 1, pp. 37-58, 2009.

[15] L. C. Ceng and J. C. Yao, "A hybrid iterative scheme for mixed equilibrium problems and fixed point problems," Journal of Computational and Applied Mathematics, vol. 214, no. 1, pp. 186201, 2008.

[16] L. Ceng, Q. H. Ansari, and S. Schaible, "Hybrid extragradientlike methods for generalized mixed equilibrium problems, systems of generalized equilibrium problems and optimization problems," Journal of Global Optimization, vol. 53, no. 1, pp. 6996, 2012.

[17] L. Ceng and J. Yao, "A relaxed extragradient-like method for a generalized mixed equilibrium problem, a general system of generalized equilibria and a fixed point problem," Nonlinear Analysis: Theory, Methods \& Applications, vol. 72, no. 3-4, pp. 1922-1937, 2010.

[18] S. Atsushiba and W. Takahashi, "Strong convergence theorems for a finite family of nonexpansive mappings and applications," Indian Journal of Mathematics, vol. 41, no. 3, pp. 435-453, 1999.

[19] L. Ceng, S. Guu, and J. Yao, "Hybrid iterative method for finding common solutions of generalized mixed equilibrium and fixed point problems," Fixed Point Theory and Applications, vol. 2012, no. 92, 19 pages, 2012.

[20] W. Takahashi and K. Shimoji, "Convergence theorems for nonexpansive mappings and feasibility problems," Mathematical and Computer Modelling, vol. 32, no. 11-13, pp. 1463-1471, 2000.

[21] V. Colao, G. Marino, and H. Xu, "An iterative method for finding common solutions of equilibrium and fixed point problems," Journal of Mathematical Analysis and Applications, vol. 344, no. 1, pp. 340-352, 2008.

[22] C. Byrne, "A unified treatment of some iterative algorithms in signal processing and image reconstruction," Inverse Problems, vol. 20, no. 1, pp. 103-120, 2004.

[23] K. Goebel and W. A. Kirk, Topics in Metric Fixed Point Theory, vol. 28 of Cambridge Studies in Advanced Mathematics, Cambridge University Press, Cambridge, UK, 1990.

[24] V. Barbu, Nonlinear Semigroups and Differential Equations in Banach Spaces, P. Noordhoff, 1976.
[25] N. Huang, "A new completely general class of variational inclusions with noncompact valued mappings," Computers \& Mathematics with Applications, vol. 35, no. 10, pp. 9-14, 1998.

[26] L. Ceng, Q. H. Ansari, M. M. Wong, and J. Yao, "Mann type hybrid extragradient method for variational inequalities, variational inclusions and fixed point problems," Fixed Point Theory, vol. 13, no. 2, pp. 403-422, 2012.

[27] L. C. Zeng, S. M. Guu, and J. C. Yao, "Characterization of $H$-monotone operators with applications to variational inclusions," Computers \& Mathematics with Applications, vol. 50, no. 3-4, pp. 329-337, 2005. 


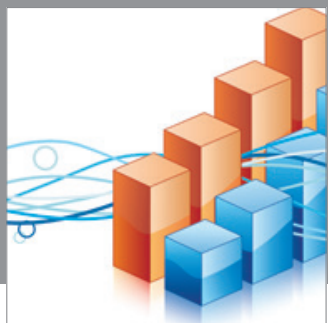

Advances in

Operations Research

mansans

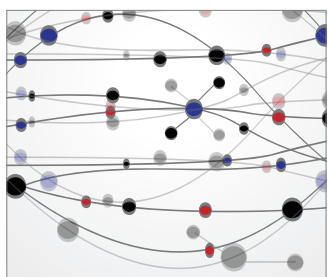

The Scientific World Journal
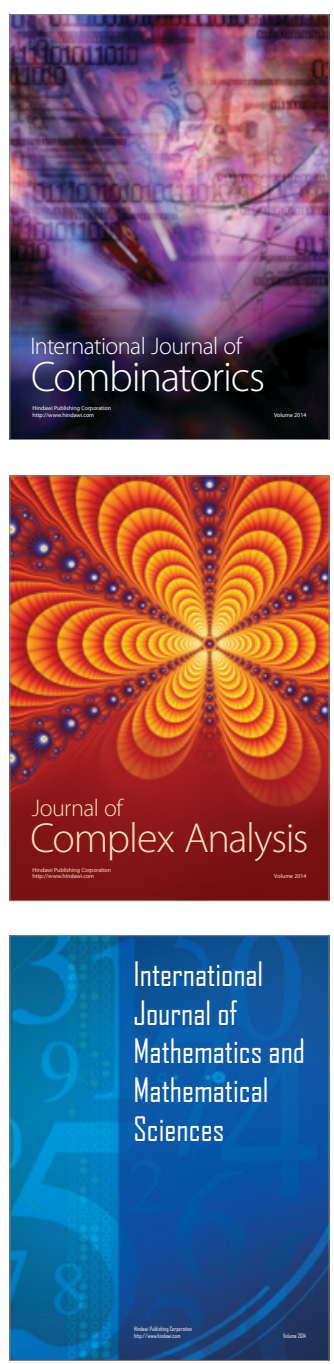
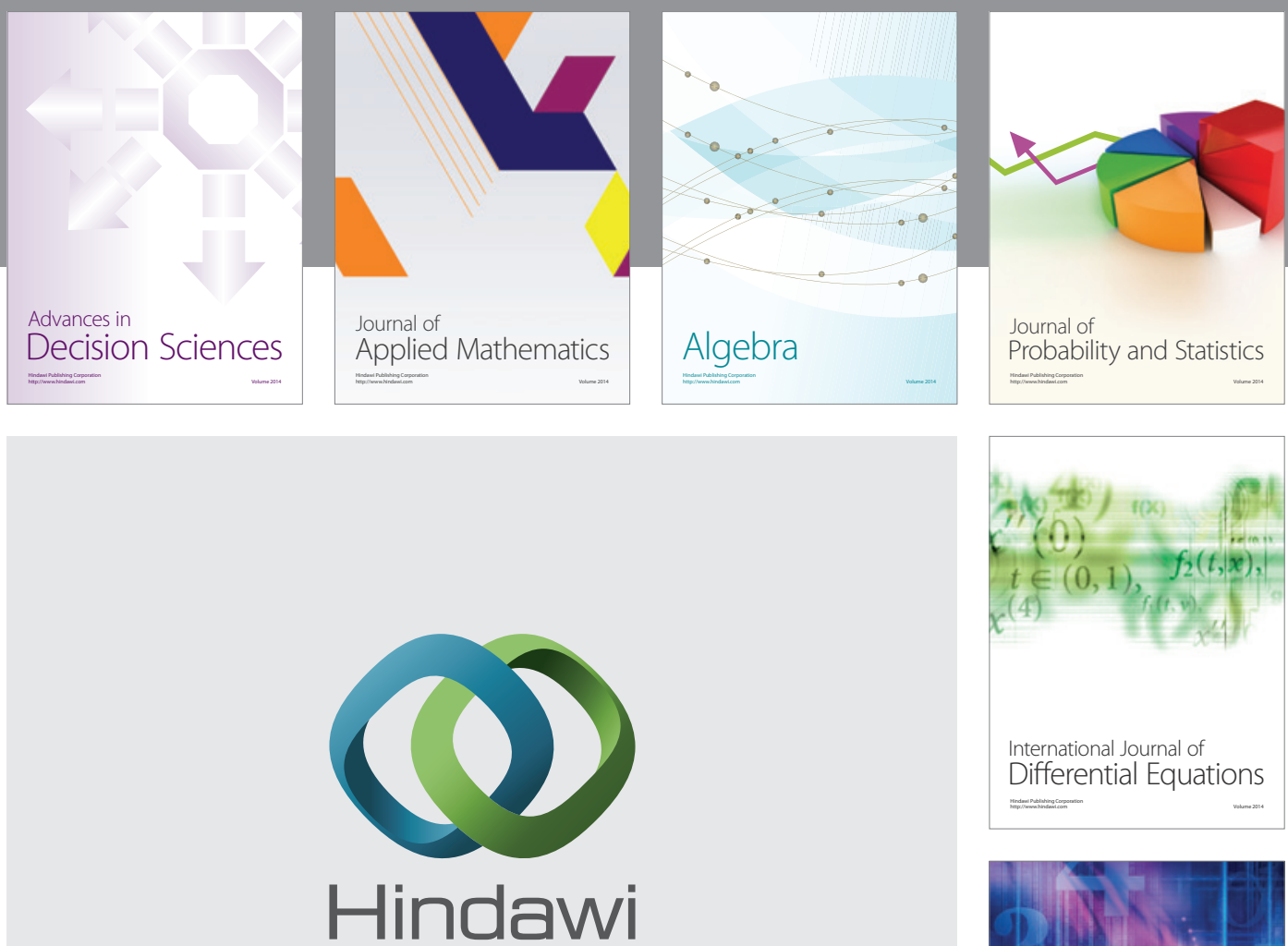

Submit your manuscripts at http://www.hindawi.com
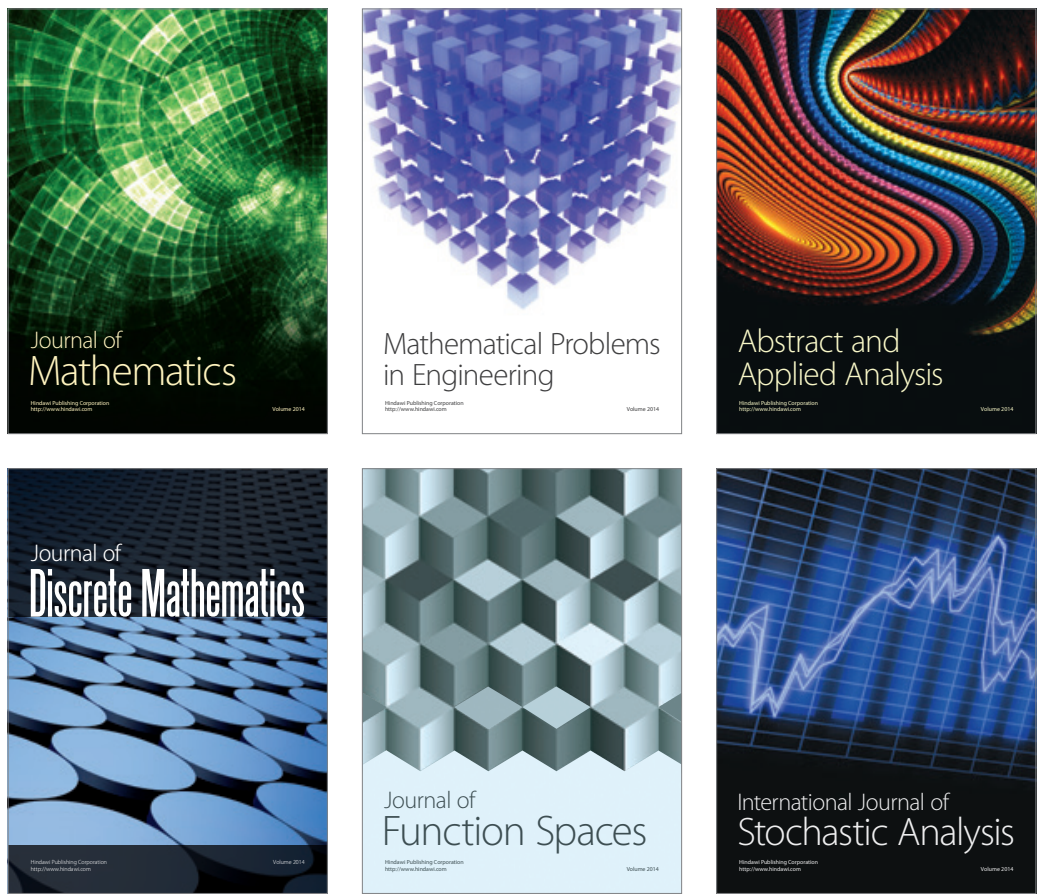

Journal of

Function Spaces

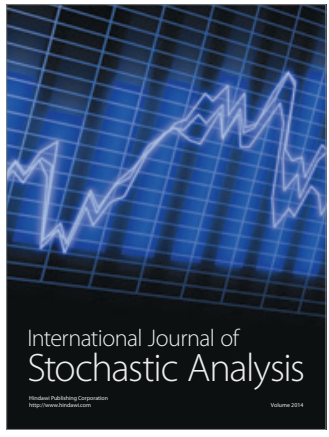

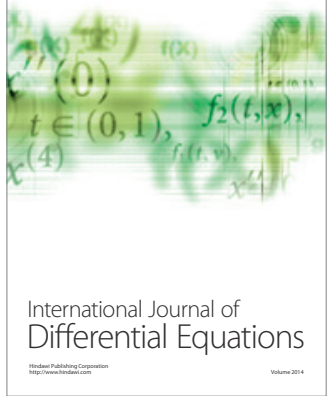
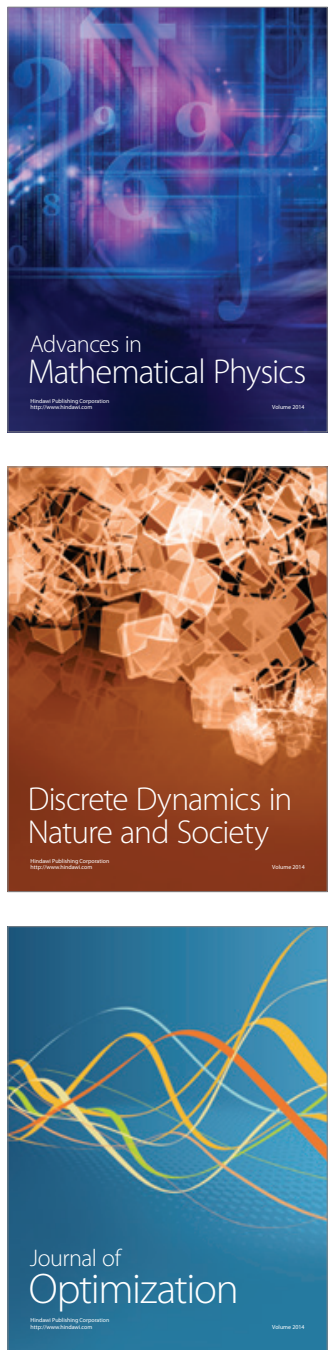